Check for updates

Cite this: RSC Adv., 2017, 7, 24070

Received 24th February 2017

Accepted 5th April 2017

DOI: $10.1039 / c 7 r a 02334 k$

rsc.li/rsc-advances

\section{Polyurethane-polylactide-based material doped with resveratrol decreases senescence and oxidative stress of adipose-derived mesenchymal stromal stem cell (ASCs)}

\author{
K. Kornicka, (D) ab D. Nawrocka, ${ }^{a}$ A. Lis-Bartos, ${ }^{c}$ M. Marędziak ${ }^{d}$ and K. Marycz ${ }^{\star a b}$
}

The aim of this study was to evaluate the influence of resveratrol (RES)-doped polyurethane (TPU)polylactide (PLA) biomaterials on the senescence and oxidative stress factor of adipose-derived stem cells (ASCs) for tissue engineering. Using a four-step method (solution preparation, solvent casting, freezing/lyophilizing, washing and drying), we fabricated TPU/PLA scaffolds doped with different RES concentrations. Then, we established the mass loss and RES release from the obtained films over time using differential scanning calorimetry and UV-Vis spectrophotometry, respectively. ASC was seeded onto the fabricated scaffolds to establish their biocompatibility and biological influence. Using fluorescence microscopy, real-time PCR, and spectrophotometric assays, the proliferation rate, oxidative stress factors, pro-apoptotic, autophagy-related and osteogenic genes expression were evaluated. The obtained data clearly indicated that the TPU/PLA biomaterial doped with $0.5 \mathrm{mmol}$ RES had the most beneficial effect on ASC, whereby it increased proliferation, reduced oxidative stress and apoptosis, and induced the expression of osteogenic genes. Fabricated by our group, innovative biomaterials were characterized by their RES release in a sustained manner. A prolonged release of RES is especially important when considering clinical application. The release of RES was most intense during the first 20 days, which may be beneficial in vivo as inflammation within damaged tissue is most intense during the first days after injury. The fabricated material directed ASC toward an osteoblast-like molecular phenotype, which suggests it could be a promising tool in regenerative medicine. The combination of the scaffold with ASC may improve the effectiveness and shorten the time for bone regeneration. These characteristics presented here make the biomaterial a promising tool in the emerging field of personalized regenerative medicine, especially in geriatrics.

\section{Introduction}

Due to rapid aging of the population worldwide, especially in developed countries, regenerative medicine and tissue engineering are two of the most rapidly developing medical fields. As estimated by the National Institute of Health (NIH), the elderly make up $30 \%$ of all patients, and this number is steadily increasing. ${ }^{1}$ Regenerative medicine and tissue engineering offer patients both stem-cell-based therapy as well as biomaterial scaffolds, which are crucial for efficient treatment. ${ }^{2-4}$ However, it has been shown that aging negatively correlates with stem cell

\footnotetext{
${ }^{a}$ Department of Experimental Biology, University of Environmental and Life Sciences, Wroctaw, Poland. E-mail: krzysztofmarycz@interia.pl; Fax: +4871320 5876; Tel: $+48713205888$

${ }^{b}$ Wroclaw Research Centre EIT+, Wroctaw, Poland

'Department of Biomaterials, AGH University of Science and Technology, Kraków, Poland

${ }^{d}$ Faculty of Veterinary Medicine, University of Environmental and Life Sciences, Wroctaw, Poland
}

multipotency, which might seriously limit their regenerative potential. In parallel with organism aging, progenitor cells lose their self-renewal, metabolic activity, and differentiation potential. Our previous work showed that with increasing patient age, mesenchymal stem cells (MSCs) lose their proliferative activity as well as their osteogenic and chondrogenic differentiation potential, which strongly limits their clinical application in geriatrics. ${ }^{5}$ Extensive oxidative stress (OS), observed in elderly patients' adipose-derived mesenchymal stromal stem cells (ASCs), can disrupt the balance between free radical activity and antioxidant defense. Abundant mitochondrial secretion of reactive oxygen species (ROS) entails a number of adverse consequences for multipotent characteristics of ASCs and thus might negatively affect their longevity as well as their regenerative properties. A decreased anabolic activity of ASCs through a limited secretion of extracellular microvesicles (ExMVs) and by creation of intercellular connections thus becomes a consequence of elevated OS. OS can have a major negative impact on ASC activity, as it has been shown that the 
regenerative ability of MSCs depends on the local delivery of growth factors and the creation of extracellular matrix formations within damaged tissue. However, accumulating evidence suggests that elevated ROS accumulation leads to a disruption of endoplasmic reticulum (ER) homeostasis, which then leads to protein oxidation, ER stress, and upregulation of a signaling pathway called the ER stress response or the unfolded protein response (UPR). ${ }^{6}$ The age-related decline of ASCs' cellular activity in key molecular chaperones and folding enzymes affects proper protein folding and the adaptive response of the UPR. Both prolonged OS and protein misfolding initiate apoptotic processes, including the upregulation of p53 gene (tumor suppressor) expression, changes in the expression of pro- and anti-apoptotic Bcl-2 family members, cytochrome C relocation, activation of caspases, chromatin condensation, and DNA fragmentation, which are strongly correlated to limitations in the regenerative process of damaged tissues in elderly patients. $^{7-9}$ The cellular antioxidative protection naturally obtained by free radical scavengers, including superoxide dismutase (SOD) activity, protects cells against molecular impairment caused by OS and improves progenitor cells and proliferative activity, and subsequently multipotency. Bearing in mind the aforementioned facts, it is fully reasonable, mainly from an elderly patient damaged tissue regeneration point of view, to assume that the precise local delivery of cells and antioxidants is more beneficial, rather than merely stimulating differentiation..$^{\mathbf{1 0 - 1 2}}$ The precise delivery of cells within damaged tissues with additional particular anti-oxidants, such as resveratrol, might result in a positive effect on the local tissue regenerative process, essentially through free radical scavenging and local cell rejuvenation. Resveratrol (trans-3,4',5-trihydroxystilbene, RES) is a plant-derived polyphenol, nonflavonoid antioxidant with various anti-aging and immunomodulatory effects. ${ }^{13}$ More and more studies have shown that RES has various beneficial effects, including increased longevity, amelioration of cardiovascular disease, improved sensitivity to insulin, and reduced cataract formation through mechanisms largely centered on pathways ultimately related to Sirtuin gene activation..$^{\mathbf{1 4}-16}$ The positive effect of RES, besides its property to scavenge free radicals, is that it can also downregulate inflammatory responses, including the inhibition of synthesis and release of proinflammatory mediators, modifications of eicosanoid synthesis, inhibition of chosen activated immune cells, or inhibition of some enzymes, such as cyclooxygenase-1 (COX-1) or cyclooxygenase-2 (COX-2), both crucial in the course of the synthesis of proinflammatory mediators. Recently, RES has been shown to promote bone healing and to reduce the risk of osteoporosis and age-related bone loss, ${ }^{17}$ due to the multiple ways of RES' action on osteoclast and osteoblast activity, including the direct increase of DNA synthesis, activation of the extracellular signal-regulated kinase (ERK) 1/2 signaling pathway, increase in ALP activity that finally affects osteoblast proliferation, differentiation, and new bone formation. ${ }^{18}$ Moreover, RES increases the gene expression of key osteogenic transcription factors, such as Runx2 or Osterix, with the simultaneous activation of AMP- activated protein kinase (AMPK), which inhibits bone resorption by acting as a negative regulator of RANKL. ${ }^{19-21}$

The incorporation of RES as an antioxidative, suppressing cellular aging factor, and inducer of the proliferative activity of elderly-patients-derived ASCs in biodegradable porous polymers seems to be a promising regenerative strategy for cellular rejuvenation. Recently, polyurethane (PU) and polymer of lactic acid (PLA) have been extensively investigated for various medical purposes, including the construction of a tube for peripheral nerve regeneration and 3D sponges for bone and/or cartilage regeneration, ${ }^{22,23}$ due to their biocompatibility and the relative ease of modulation of their microstructure and mechanical properties. It was recently demonstrated that PU/ PLA-based biomaterials released nontoxic degradation products, which did not reduce cellular viability. Our recent findings indicated that 3D sponges composed with the PU/PLA support adhesion of ASCs in vitro maintain their proper morphology and behavior, and furthermore this enhances their proliferative activity. ${ }^{23}$ Furthermore, we found, that PU/PLA-based scaffolds reduce intracellular ROS activity with the concurrent elevated secretion of SOD in human ASCs. Additionally, it was found, that ASCs cultivated onto PU/PLA-based scaffolds exhibited an upregulation of BMP-2/4, Sox9, and type I collagen transcripts at the mRNA level.

In our current research, we fabricated PU/PLA blends, functionalized with RES, for reducing the OS of elderly-patientsderived ASCs. We found that the fabricated biomaterial had beneficial effects on ASC's viability and proliferative potential with a concurrent decrease in OS and in the induced activity of free radical scavengers, including SOD.

\section{Materials and methods}

\section{Polyurethane/polylactide/resveratrol film preparation}

\section{Polymer film preparation}

Reagents. Pure polyurethane/polylactide (TPU/PLA) blend and TPU/PLA blends with different resveratrol (RES) content were tested in this research. Thermoplastic polyurethane "Ellastolan ${ }^{\circledR}$ A12P000-INTiBS" (TPU) was purchased from the Polish Academy of Sciences (PAN) (Wroclaw, Poland). Rigid polylactide "PURASORB®" (PLA), consisting of 20\% poly-DLlactide and $80 \%$ poly-L-lactide, was obtained from PURACbiochemby (Gorinchem, The Netherlands). Resveratrol was purchased from Sigma-Aldrich. Sterile distilled water, $\mathrm{N}, \mathrm{N}$ dimethylformamide (DMF), and methylene iodide were obtained from Sigma-Aldrich (Poznan, Poland).

Step I: solution preparation. Polyurethane/polylactide solution was prepared by dissolving $10 \% \mathrm{w} / \mathrm{w}$ of mixed polymer grain in dimethylformamide (DMF). The resveratrol powder was dissolved with different concentrations in distilled water at $40{ }^{\circ} \mathrm{C}$ and stirred in a glass bottle. Then, resveratrol solution was added to the polymer solution and the mixed solution was stirred for $1 \mathrm{~h}$. The mixed solutions were prepared by adding resveratrol water solution to the polymer solutions with different concentrations (Table 1).

Step II: solvent casting. Polymer pre-films were formed by the standard solvent casting method. The prepared TPU/PLA/ 
Table 1 Mass ratio of polymers and molar concentration of resveratrol

\begin{tabular}{llll}
\hline $\begin{array}{l}\text { Sample } \\
\text { composition }\end{array}$ & $\begin{array}{l}\text { Polyurethane } \\
\text { mass }[\mathrm{g}]\end{array}$ & $\begin{array}{l}\text { Polylactide } \\
\text { mass }[\mathrm{g}]\end{array}$ & $\begin{array}{l}\text { Resveratrol } \\
\text { concentration } \\
\mathrm{mmol} \mathrm{ml}^{-1}\end{array}$ \\
\hline TPU/PLA 8/2 & 8.0 & 2.0 & - \\
TPU/PLA 8/2 + 0.1 & 8.0 & 2.0 & 0.1 \\
TPU/PLA 8/2 0.5 & 8.0 & 2.0 & 0.5 \\
TPU/PLA 8/2 + 1.0 & 8.0 & 2.0 & 1.0
\end{tabular}

resveratrol solution in DMF/distilled water was immediately cast into Petri dishes. The Petri dishes were kept in an air atmosphere at room temperature for $1 \mathrm{~h}$.

Step III: freezing/freeze-drying process. The polyurethane/ polylactide/resveratrol pre-film was frozen at $-65{ }^{\circ} \mathrm{C}$ immediately after its formation for $24 \mathrm{~h}$. The frozen TPU/PLA pre-films were then placed in a freeze drier apparatus and lyophilized for $24 \mathrm{~h}$ to generate thin polymer films with variable resveratrol concentrations.

Step IV: washing and drying. After the lyophilization process, the polymer TPU/PLA/resveratrol films were washed in distilled water to remove any remaining solvent and then dried in an air atmosphere.

A schematic illustration showing the fabrication of the TPU/ PLA/resveratrol film is shown in Fig. 1.

\section{Evaluation of the physical and chemical properties of the TPU/ PLA/resveratrol films}

In order to recognize the changed behavior of the TPU/PLA blends with various amounts of resveratrol, some characterization tests were carried out.
Contact angle measurement. The contact angle $\Theta$ of the prepared polyurethane/polylactide and TPU/PLA/resveratrol thin films was measured using drop shape analysis. The analysis was performed using a DSA 10 Mk2 Goniometer (Kruss, Germany) as the measuring device. The measurement was taken using ultra-pure water, provided by PURE LAB UHQ Model apparatus (Vivendi Water Solution), and diiodomethane (Sigma-Aldrich). The volume of the measurement drops for the water and methylene iodide was $0.2 \mu \mathrm{l}$. Measurements were performed at standard temperature, i.e., $25{ }^{\circ} \mathrm{C}$. The data presented herein are the average of ten measurements $( \pm$ standard deviation).

Profilometer analysis. The roughness of the TPU/PLA/ resveratrol films was measured using a profilometer (Hommelwerke, Germany), equipped with a cone-shaped diamond tip (radius of $5 \mu \mathrm{m}$ ) and the velocity of moving cone was $0.5 \mathrm{~mm}$ $\mathrm{s}^{-1}$. The roughness of ten different spots on both sides (top and bottom) of each sample was measured and the surface average roughness $\left(R_{\mathrm{a}}\right)$ was calculated. The presented results herein are the average of ten measurements ( \pm standard deviation).

Tensile test. Young's moduli $(E)$ value of the polymer films $(60 \times 6 \times 1 \mathrm{~mm})$ were measured using a universal testing machine (Zwick 1345, Germany) equipped with a $1 \mathrm{kN}$ load cell. The blend sample length between the clamps was $40 \mathrm{~mm}$ and the clamps speed was $50 \mathrm{~mm} \mathrm{~min}^{-1}$. The mechanical properties were determined in the dry state in room temperature. The data presented herein are the average of ten measurements ( \pm standard deviation).

Thermal analysis: thermogravimetry (TGA)/differential scanning calorimetry (DSC). The thermal degradation and thermal behavior of the TPU/PLA and TPU/PLA/resveratrol film were measured via thermogravimetric analysis (TGA) and

\section{The process of polymer film preparation}

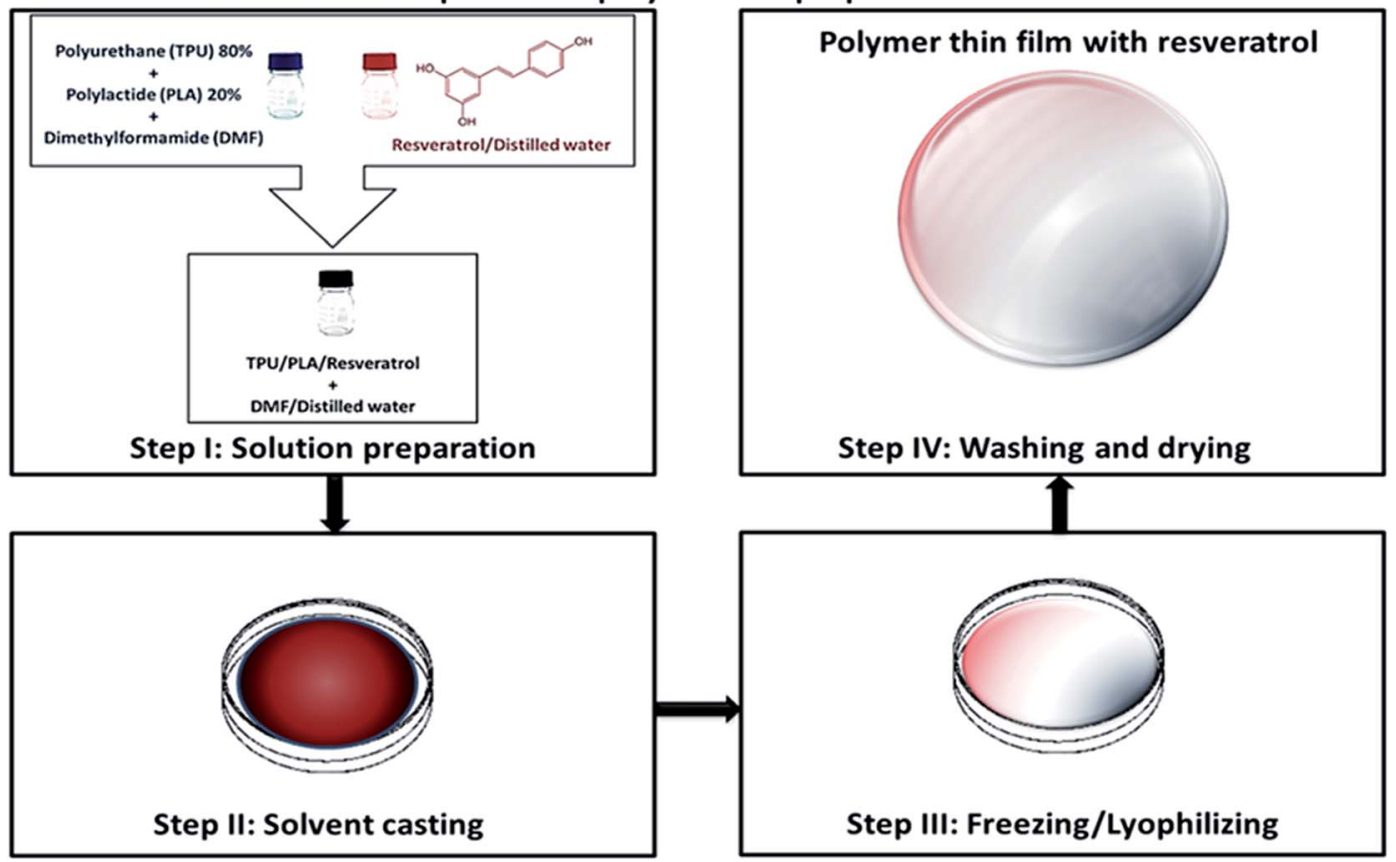

Fig. 1 Schematic showing the fabrication of the TPU/PLA/resveratrol film by the solvent casting and freeze-drying technique. 
differential scanning calorimetry (DSC) (NETZSCH STA 449 F3, Germany), respectively, under a nitrogen atmosphere. The samples (sample mass: $10 \mathrm{mg}$ ) were heated at a rate of $10{ }^{\circ} \mathrm{C}$ $\min ^{-1}$ from $20{ }^{\circ} \mathrm{C}$ to $500{ }^{\circ} \mathrm{C}$. Fig. 4 and 5 presents the TGA and DSC analyses of the studied polymer films. From the TGA curves, the thermal degradation characteristics, such as the onset degradation $\left(T_{\mathrm{d}}\right)$, temperature at maximum rate degradation ( $\left.T_{\text {max. }}\right)$, and temperatures of different mass losses, can be determined.

In vitro release studies. The in vitro release of resveratrol from the polyurethane/polylactide blends was performed using a UV-Vis spectrophotometer and in $100 \mathrm{ml}$ distilled water maintained at $37 \pm 1{ }^{\circ} \mathrm{C}$. The TPU/PLA circle samples ( $\varnothing=14 \pm$ $1 \mathrm{~mm}$ ) were placed in the dissolution PP vessels and the paddle was adjusted to rotate at $500 \mathrm{rpm}$. Samples of $10 \mathrm{ml}$ were withdrawn at predetermined intervals, filtered through a membrane filter of $0.45 \mu \mathrm{m}$ pore size, and then spectrophotometrically measured for resveratrol.

In vitro degradation tests in distilled water. The in vitro degradation study of TPU/PLA and TPU/PLA/resveratrol films was carried out in distilled water at $37 \pm 1{ }^{\circ} \mathrm{C}$. The polymer films were each weighed and then immersed in distilled water. Ten pre-wetted thin films of each type were placed in $30 \mathrm{ml}$ PP tubes containing $20 \mathrm{ml}$ distilled water. The samples were stored in a laboratory incubator at $37{ }^{\circ} \mathrm{C}$ for up to 20 weeks. At weekly intervals, the mass of dried samples and the $\mathrm{pH}$ of the distilled water were measured using a $\mathrm{pH}$ meter and then replaced with fresh water.

\section{In vivo assays with ASCs}

Ethics statement. The study was approved by the Local Bioethics committee of the Wroclaw Medical School (registry number KB-177/2014). Written consent for using the samples for research purposes was obtained from all patients prior to surgery.

Cells and culture. Human mesenchymal stem/stromal cells were isolated from adipose tissue. Patients' fat biopsies were obtained during total hip arthroplasty. Each tissue sample was placed into sterile Hank's Balanced Salt Solution (HBSS). The adipose tissues were washed extensively with HBSS containing $1 \%$ antibiotic-antimitotic solution (penicillin/streptomycin/ amphotericin b). After removal of the blood vessels, the tissues were cut into small pieces with surgical scissors. The samples were transferred into sterile tubes and digested with collagenase type I enzyme $\left(1 \mathrm{mg} \mathrm{m}{ }^{-1}\right)$. Tissues samples were incubated for $40 \mathrm{~min}$ at $37{ }^{\circ} \mathrm{C}$. The tissue homogenate was centrifuged for $10 \mathrm{~min}$ at $1200 \times \mathrm{g}$ at room temperature. After centrifugation, the supernatant was discarded and the pellet resuspended in Dulbecco's Modified Eagle's Medium (DMEM, Sigma-Aldrich) with nutrient F-12 Ham, 10\% Fetal Bovine Serum (FBS, Sigma-Aldrich), and 1\% antibiotic solution and transferred to a culture flask. The primary culture of humanadipose-derived mesenchymal stromal cells (hASCs) was designated "passage 0 " and was cultured at $37^{\circ} \mathrm{C}$ in a humidified $5 \% \mathrm{CO}_{2}$ to $95 \%$ air atmosphere. The medium was changed every three days until the cells reached approximately $80 \%$ confluence. Adherent cells were detached from the flask using TrypLE ${ }^{\text {TM }}$ Express (Life Technologies, Poland). The cells were passaged three times before introducing them into the experiments. The purity of the mesenchymal stem cells was proved by FACS by the absence of the hematopoietic markers (CD34 and CD45), the presence of mesenchymal markers (CD90, CD73b, $\mathrm{CD} 44, \mathrm{CD} 29)$, and their ability to differentiate chondroblasts, osteoblasts, and adipocytes.

The test cells were maintained in 24-well plates and inoculated at a concentration of $30 \times 10^{3}$ cells per well. The media were changed every two days. Chondrogenic and osteogenic stimulation was conducted for 21 days, whereas the cells were cultured in adipogenic medium for 14 days.

Multipotency assay of the ASCs. Osteogenic, chondrogenic, and adipogenic differentiation of the cells were induced using commercial kits (STEMPRO ${ }^{\circledR}$ Osteogenesis Differentiation Kit and STEMPRO ${ }^{\circledR}$ Adipogenesis Differentiation Kit, both Life Technologies) in accordance with the manufacturers' protocols. In order to perform the tests, the cells were seeded in a 24-well plate at an initial density of $2 \times 10^{4}$ and the media were changed every two days. Experiments were carried out simultaneously, each in triplicate. The stimulation of osteoand chondrogenesis lasted 21 days, while stimulation toward the adipogenic lineage lasted for 14 days. Cultures expanded in standard growth medium were used as a control to allow for establishing the differentiation effectiveness. Multilineage differentiation was confirmed at 2 weeks postinduction by cell staining. To evaluate the results of the differentiation process, the cells were fixed with $4 \%$ ice-cold paraformaldehyde (PFA) and specific stainings were performed. The extracellular mineralized matrix was visualized with Alizarin red dye, while the formation of proteoglycans was confirmed by Safranin O. Intracellular lipid droplets were stained red with Oil Red O. Cells were observed under an inverted microscope (AxioObserverA1, Zeiss) and photographs were acquired using a Cannon PowerShot digital camera.

Cell proliferation assay. Cell viability was determined with the resazurin-based assay kit (Sigma-Aldrich) performed according to the manufacturer instructions. The viability of the cells was estimated at the $2 \mathrm{nd}, 5 \mathrm{th}, 7 \mathrm{th}, 12 \mathrm{th}, 14 \mathrm{th}$, and 16 th days of the experiment. The absorbance of the samples was measured spectrophotometrically (Spectrostar Nano, BMG Labtech) at a reference wavelength of $690 \mathrm{~nm}$ and subtracted from the $600 \mathrm{~nm}$ peak. The amount of cells was measured based on a standard curve performed during the test. Based on the absorbance, the proliferation factor and population doubling time were calculated according to the previously described method.

The morphology, ultrastructure, growth pattern, and senescence analysis of hASCs cultured on the investigated biomaterials. The morphology of the cells, cellular composition, and culture growth patterns were evaluated using an inverted, fluorescence microscope (AxioObserverA1, Zeiss) and a scanning electron microscope (SEM; EVO LS15, Zeiss). In order to perform the analysis, the cells were fixed in $4 \%$ paraformaldehyde for $30 \mathrm{~min}$ at room temperature. Washing of the 
samples was performed between every step of the procedure. Before staining, the cell membranes were permeabilized with $0.1 \%$ Triton X-100 prepared in the washing solution. The mitochondria were stained using a rhodamine-based dye, MitoRed (Sigma-Aldrich, Germany), whereas the nuclei were stained using diamidino-2-phenylindole (DAPI). The cytoskeleton was dyed using atto-488-labeled phalloidin. The procedures involving fluorescence staining were performed in accordance with the manufacturers' instructions and methods described previously. To observe the cells with SEM, the samples were washed with distilled water and dehydrated by passing them through a graded series of ethanol-water mixtures (from $50 \%$ to $100 \%$, every $10 \%$ ). The morphology of the cells was evaluated using an SE1 detector, at $10 \mathrm{kV}$ filament tension (SEM, Zeiss Evo LS 15).

The amounts of viable and dead cells were evaluated with the Cellstain Double Staining Kit (Sigma-Aldrich). Viable cells were stained with calcein-AM and emitted green fluorescence, whereas dead cells' nuclei were stained orange with propidium iodide. Cells were then observed using fluorescence microscopy (Zeiss, Axio Observer A.1). Staining for caspase 3 was performed using anti-caspase 3 antibodies (Anti-Caspase 3 active antibody produced in rabbit, Sigma-Aldrich).

Assessment of oxidative stress and senescence. Prior to assessing the level of oxidative stress, the cells were cultured in normal DMEM growth medium without phenol red. The superoxide dismutase (SOD) activity was measured with a commercially available SOD Assay kit (Sigma-Aldrich, Germany), whereas the level of nitric oxide (NO) was estimated using a Griess reagent kit (Life Technologies). The production of ROS was determined by measuring H2DCF-DA (Life Technologies). All the procedures were performed in duplicate, according to the manufacturers' instructions.
Analysis of gene expression: real-time reverse transcription polymerase chain reaction (qRT-PCR). On the 7 th day of the experiment, the cells were homogenized using $1 \mathrm{ml}$ TRI reagent. Total RNA was isolated using the phenol-chloroform method as previously described by Chomczynski and Sacchi. The quality and quantity of isolated total RNA were determined using a nano-spectrometer (WPA Biowave II). Genomic DNA digestion and cDNA synthesis were performed using the PrimeScript kit (Takara, Clontech). For each reaction, $150 \mathrm{ng}$ of total RNA was used. Both processes were performed in accordance with the manufacturers' instructions using a T100 Thermal Cycler (BioRad).

The qRT-PCR reactions were performed using a CFX ConnectTM Real-Time PCR Detection System (BioRad). The reaction mixture contained $2 \mu \mathrm{l}$ of cDNA in a total volume of $20 \mu \mathrm{l}$ using the SensiFast SYBR \& Fluorescein kit (Bioline). The concentration of primers in each reaction equaled $500 \mathrm{nM}$; the primer sequences used in the individual reactions are listed in Table 2. Relative gene expression analysis $\left(Q_{\mathrm{n}}\right)$ was calculated in relation to the GAPDH housekeeping gene.

Statistical analysis. All the results are expressed herein as the mean \pm standard deviation (SD). Statistical significance was determined using the un-paired $t$-test or one-way ANOVA with Dunnett post hoc test (Prism 5.04, GraphPad Software, CA, USA). $p<0.05$ was considered statistically significant.

\section{Results}

\section{Contact angle measurements}

The wettability of the TPU/PLA-based samples was investigated by measuring the contact angle of water and diiodomethane dropped on the surface of treated region using a contact angle analyzer, and ten evaluations were averaged. Table 3 shows the

Table 2 Sequence of primers used in $\mathrm{qRT}^{-\mathrm{PCR}^{a}}$

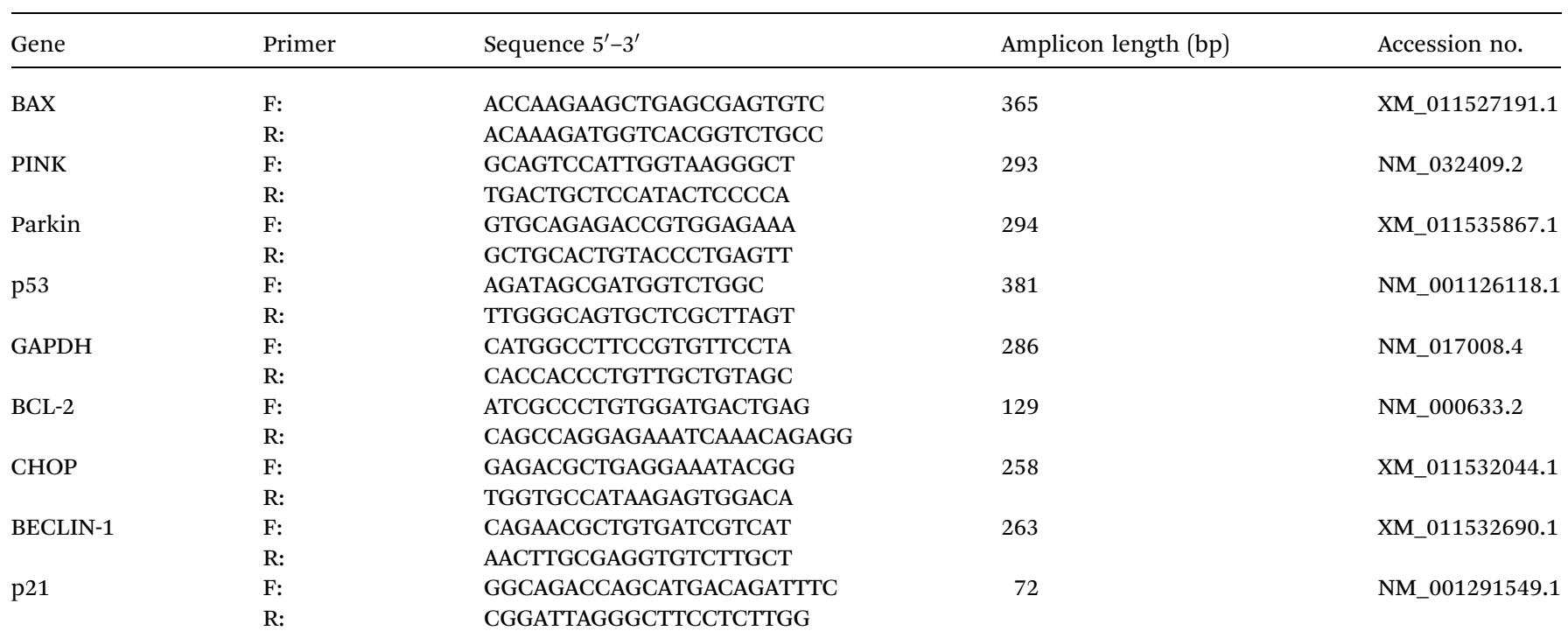

${ }^{a}$ BAX - BCL2-associated X protein; PINK - PTEN induced putative kinase 1; Parkin - Parkin ligase; p53 - tumor suppressor p53; GAPDH glyceraldehyde 3-phosphate dehydrogenase; BCL-2 - B-cell lymphoma 2; CHOP - transcription factor C/EBP homologous protein; BECLIN-1; p21 - cyclin-dependent kinase inhibitor $1 \mathrm{~A}$. 
Table 3 The results of the contact angle measurements for the TPU/ PLA and TPU/PLA/resveratrol films in two measurement liquids

\begin{tabular}{llllll}
\hline & \multicolumn{2}{l}{$\begin{array}{l}\text { Water contact } \\
\text { angle } \Theta_{\mathrm{w}}\left[^{\circ}\right]\end{array}$} & & \multicolumn{2}{l}{$\begin{array}{l}\text { Diiodomethane contact } \\
\text { angle } \Theta_{\mathrm{D}}\left[^{\circ}\right]\end{array}$} \\
\cline { 2 - 3 } Sample code & Top & Bottom & & Top & Bottom \\
\hline TPU/PLA 8/2 & $82.9 \pm 1.9$ & $85.3 \pm 1.3$ & & $45.3 \pm 1.8$ & $57.1 \pm 0.9$ \\
TPU/PLA 8/2 +0.1 & $83.5 \pm 1.4$ & $86.6 \pm 1.2$ & & $34.2 \pm 1.3$ & $53.7 \pm 1.4$ \\
TPU/PLA 8/2 + 0.5 & $81.2 \pm 1.8$ & $84.7 \pm 0.8$ & & $43.9 \pm 1.9$ & $56.2 \pm 1.8$ \\
TPU/PLA 8/2 +1.0 & $79.8 \pm 1.5$ & $83.9 \pm 1.7$ & & $36.9 \pm 1.7$ & $49.5 \pm 1.1$
\end{tabular}

contact angle of water and diiodomethane for all the TPU/PLA samples with and without resveratrol. Generally, the contact angle decreased with increasing the resveratrol concentration. The water contact angle on the bottom surface of the examined materials was higher than on the top surface. Table 3 shows that the diiodomethane contact angle for all the samples was lower than the noticed water contact angle on the same surface. The TPU/PLA/RES films showed decreased hydrophobicity (increasing hydrophilicity). In addition, the lower value of the contact angle for all the TPU/PLA/RES compositions and for both the measurement liquids suggests an improvement in the wettability, which is beneficial for the cell culture.

\section{Surface characterization and roughness measurements}

The average roughness $\left(R_{\mathrm{a}}\right)$, a frequently used and wellestablished roughness parameter, is commonly inadequate for describing the specific character of biomaterial roughness. Profilometry was thus used to assess the surface topography of the TPU/PLA blends. Ten different regions for each sample were measured, the average roughness values are presented in Table 4 . The average surface roughness $\left(R_{\mathrm{a}}\right)$ of the TPU/PLAbased film was around $80 \mu \mathrm{m}$. Generally, for the bottom side of the samples (flatter surface), the average roughness value was smaller than for the top side of the materials (becomes rougher).

\section{Tensile tests}

The tensile modulus of the TPU/PLA compositions decreased with the increasing resveratrol concentration, which indicated that the stiffness of the composition decreased with filler loading. The relationship between the resveratrol amount and the tensile modulus for the TPU/PLA/resveratrol formulation is

Table 4 The results of the roughness analysis for the TPU/PLA blend and TPU/PLA/resveratrol films

\begin{tabular}{lll}
\hline & \multicolumn{2}{l}{ Roughness $R_{\mathrm{a}}[\mu \mathrm{m}]$} \\
\cline { 2 - 3 } Sample code & Top & Bottom \\
\hline TPU/PLA 8/2 & $85.3 \pm 1.3$ & $82.9 \pm 1.9$ \\
TPU/PLA 8/2 +0.1 & $86.6 \pm 1.2$ & $83.5 \pm 1.4$ \\
TPU/PLA 8/2 +0.5 & $84.7 \pm 0.8$ & $81.2 \pm 1.8$ \\
TPU/PLA 8/2 +1.0 & $79.8 \pm 1.5$ & $83.9 \pm 1.7$
\end{tabular}

presented in Table 5. For the TPU/PLA blend containing $1.0 \mathrm{mM}$ $\mathrm{ml}^{-1} \mathrm{RES}$, the modulus fell to $15.21 \mathrm{MPa}$ as compared to 19.16 MPa for the pure TPU/PLA blend. The percentage elongation at break increased with the increase in the RES content.

\section{In vitro degradation study}

Fig. 2 shows the degradation rate of the TPU/PLA films and TPU/ PLA/resveratrol films with the degradation time at $37^{\circ} \mathrm{C}$. The mass loss of TPU/PLA/resveratrol was faster than the mass loss of TPU/PLA films without resveratrol at the same temperature and time, but was similar for the TPU/PLA blend with $0.1 \mathrm{mM}$ $\mathrm{ml}^{-1}$ of resveratrol. The results indicated that the degradation rate of the films increased with increasing the resveratrol amount. Other results (unpresented) showed that the $\mathrm{pH}$ of the degradation media decreased during the first weeks of degradation time, but after 7 weeks increased. The $\mathrm{pH}$ of the degradation media surrounding all the samples was analogous. A faster degradation of the TPU/PLA blend and lower $\mathrm{pH}$ values of the degradation media in the opening weeks corresponded with a degradation of polylactide, and acidic degradation products accumulating in the media.

\section{Resveratrol in vitro release study}

An in vitro resveratrol release study was performed for all the TPU/PLA/resveratrol formulations that passed the stability tests. Resveratrol release from TPU/PLA blends was investigated by immersing the samples in distilled water, where the amount of released RES was measured via UV-Vis spectroscopy.

Table 5 Tensile $(E)$ modulus of TPU/PLA films with and without resveratrol

\begin{tabular}{ll}
\hline Sample code & $E$-Modulus [MPa] \\
\hline TPU/PLA 8/2 & $19.16 \pm 1.18$ \\
TPU/PLA 8/2+0.1 & $16.74 \pm 2.37$ \\
TPU/PLA 8/2 0.5 & $17.55 \pm 2.08$ \\
TPU/PLA 8/2 1.0 & $15.21 \pm 1.06$
\end{tabular}

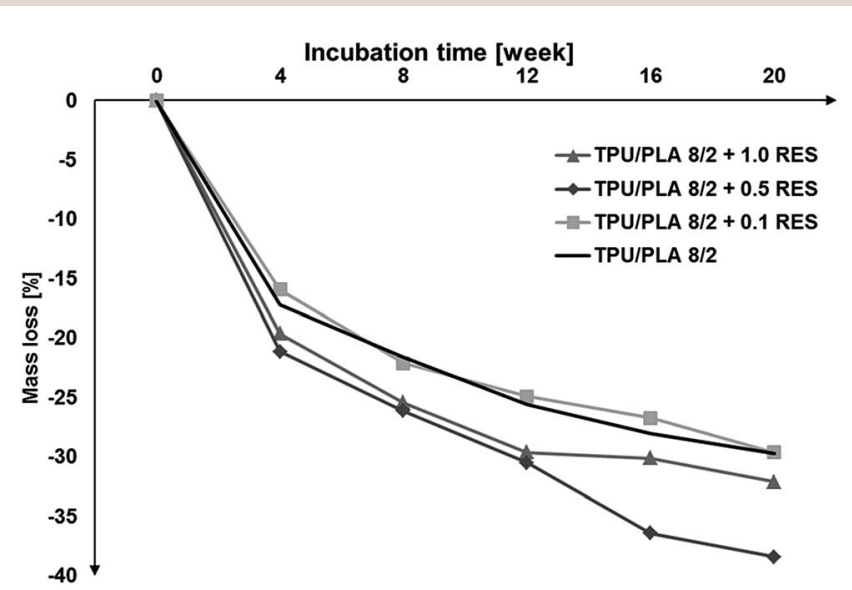

Fig. 2 Change of mass of the TPU/PLA and TPU/PLA/resveratrol films with different initial resveratrol amounts degraded in water at $37^{\circ} \mathrm{C}$. 
Measurements were taken at moderately short intervals (every day) during the first 3 days to monitor the initial burst release. The final release profile of each sample was plotted as the cumulative percentage of RVT released versus time (Fig. 3). The amount of resveratrol released in the first day ranged from $0.1 \%$ to $1 \%$. There was a similar resveratrol release pattern for all the TPU/PLA/RES compositions after $48 \mathrm{~h}$. The dissolution profiles of resveratrol from TPU/PLA with 0.1 and $0.5 \mathrm{mM} \mathrm{ml}^{-1}$ were very similar.

\section{Thermal analysis}

Fig. 4 show the thermal degradation behavior of $10 \mathrm{mg}$ samples of each of the TPU/PLA samples heated at $10 \mathrm{~K} \mathrm{~min}^{-1}$ under a nitrogen atmosphere in TGA/DSC equipment at normal pressure. Fig. 4 shows the normalized decomposition plots for the TPU/PLA blends. Fig. 4 and 5 show similar profiles, with decomposition beginning at approximately $235{ }^{\circ} \mathrm{C}$ and extending to $390{ }^{\circ} \mathrm{C}$, as clearly observed from the DSC curves. The maximum decomposition rates were observed at about 360$370{ }^{\circ} \mathrm{C}$. In the case of the pure TPU/PLA blends, the shape of the curve in Fig. 4 shows a trivial difference. The main decomposition occurred between $290{ }^{\circ} \mathrm{C}$ and $420{ }^{\circ} \mathrm{C}$. This sample lost

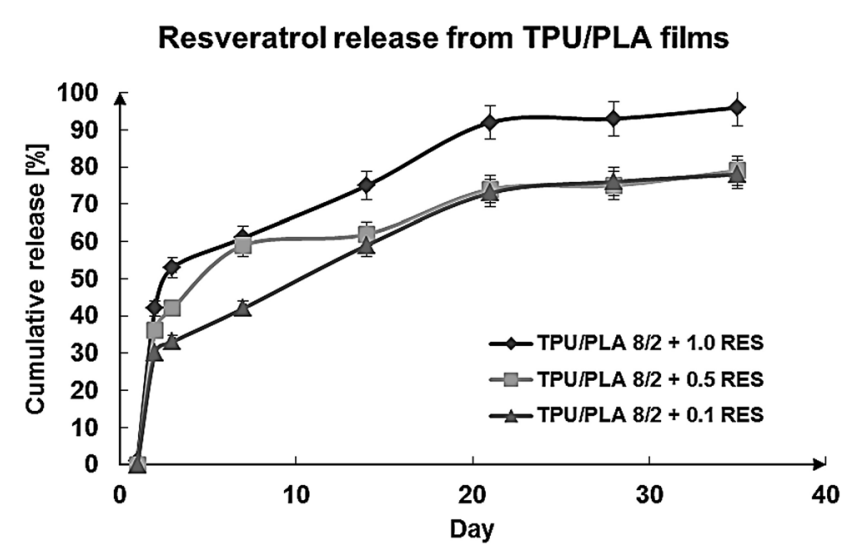

Fig. 3 Dissolution profiles of resveratrol from the TPU/PLA blend prepared using different resveratrol amounts.

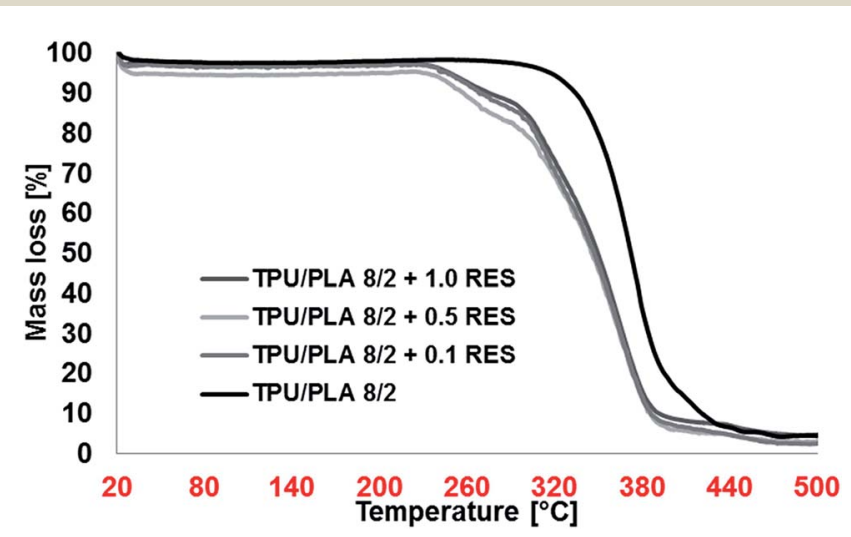

Fig. 4 TGA curves in nitrogen atmosphere for: pure TPU/PLA blend (black line), TPU/PLA with resveratrol (gray lines): resveratrol concentration: $0.1,0.5$, and $1.0 \mathrm{mM} \mathrm{ml}^{-1}$.

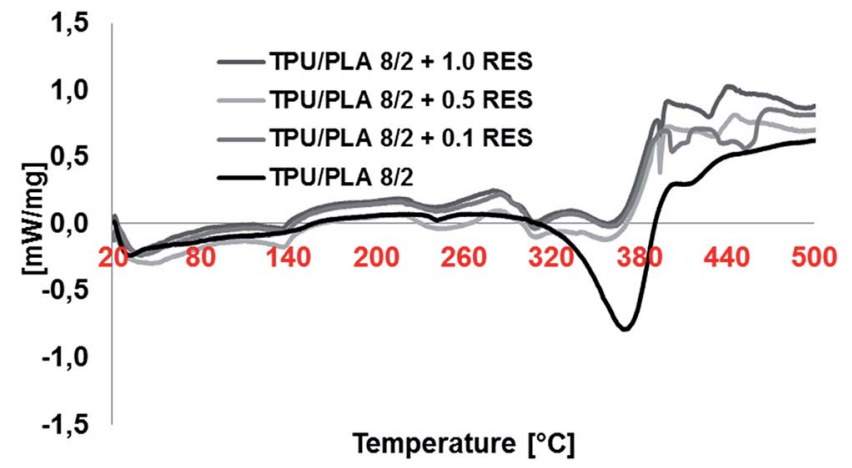

Fig. 5 DSC curves in nitrogen atmosphere for: pure TPU/PLA blend (black line), TPU/PLA with resveratrol (gray lines): resveratrol concentration: $0.1,0.5$, and $1.0 \mathrm{mM} \mathrm{m}^{-1}$.

a smaller weight percentage $(50 \%)$ at $370{ }^{\circ} \mathrm{C}$, corresponding to the maximum rate of decomposition. Furthermore, there are a few small peaks in the DSC curve of the TPU/PLA sample. The thermal analysis results showed that the decomposition behavior of the samples with resveratrol are similar and more complex than that of the TPU/PLA sample, and thus it is likely that the chemical nature of the TPU/PLA/RES films is different from that of the sample without resveratrol.

The hASCs: FACS analysis, immunophenotyping, and multipotency test

Flow cytometry (FACS) confirmed the presence of the following hASCs surface markers: CD44, CD73, CD105, and CD90 (Fig. 6A). At the same time, the presence of hematopoietic markers, i.e., CD45 and CD34, was excluded. Moreover, the osteogenic, adipogenic, and chondrogenic of the hASCs revealed the formulation of osteo- and chondro-nodules as well as lipid droplets. The functionality of osteo, chondro, and adipo differentiation was confirmed histochemically with a positive reaction to Safranin $\mathrm{O}$ (glycosaminoglycans), Alizarin red (osteoblasts), and Oil Red O staining (lipid droplets) (Fig. 6B). The stained area was calculated using Image J. Moreover, ELISA tests for collagen 1 , collagen 2 , and adiponectin were performed in accordance with the manufacturer's instructions (EIAB).

\section{Cell morphology}

Human ASCs cells presented diverse morphology regarding the type of material. Phalloidin/DAPI staining revealed that the cells did not create colonies and their actin cytoskeleton was significantly reduced (Fig. 7). In the pure TPU/PUA control, the cells were mostly viable, and the quantity of death cells was insignificant. Phalloidin/DAPI staining revealed the proper bipolar, elongated shape of ASCs and the presence of small, elongated colonies, but created dense aggregates after 5 days of culture.

\section{Proliferation assay and oxidative stress factors analysis}

During the 5 day assessment of the proliferative potential of the hASCs, we observed diverse proliferative activity regarding the quantity of resveratrol present in the biomaterial. The highest 
A

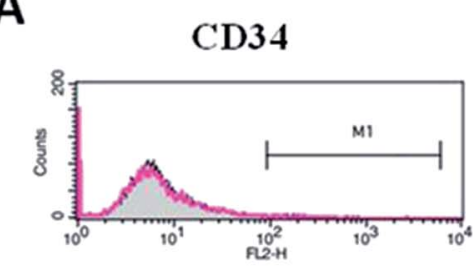

CD73

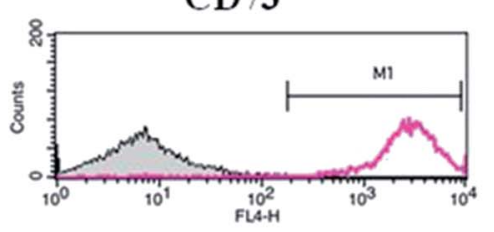

B

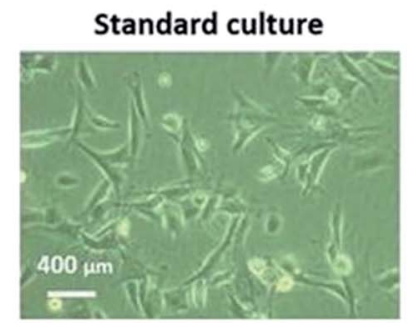

C

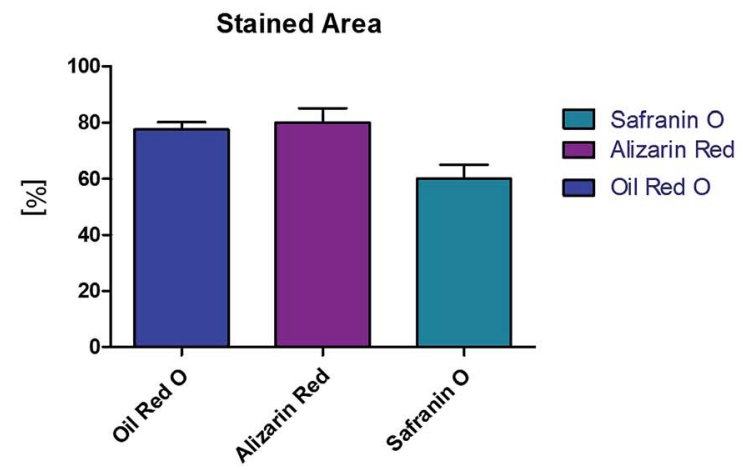

CD44

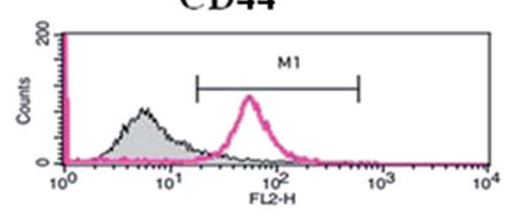

CD90

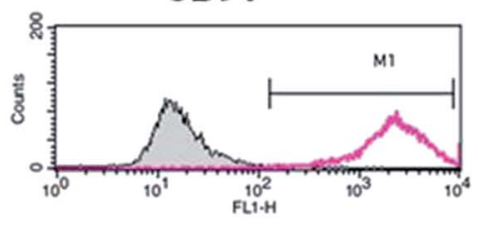

CD45

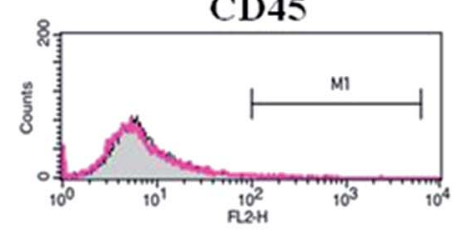

CD105

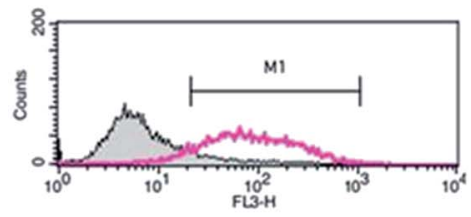

Chondrogenic stimulation Osteogenic stimulation

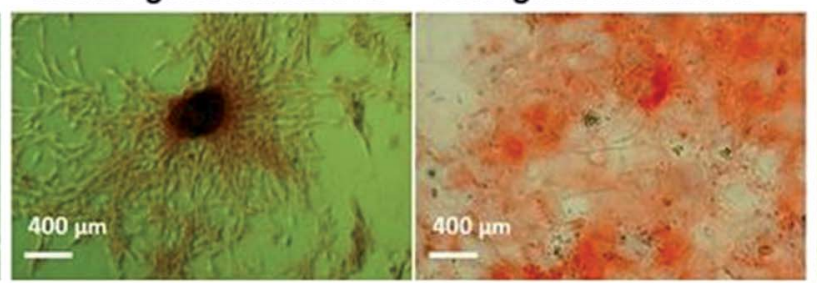

Adipogenic stimulation

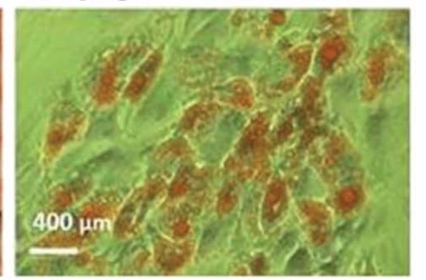

D

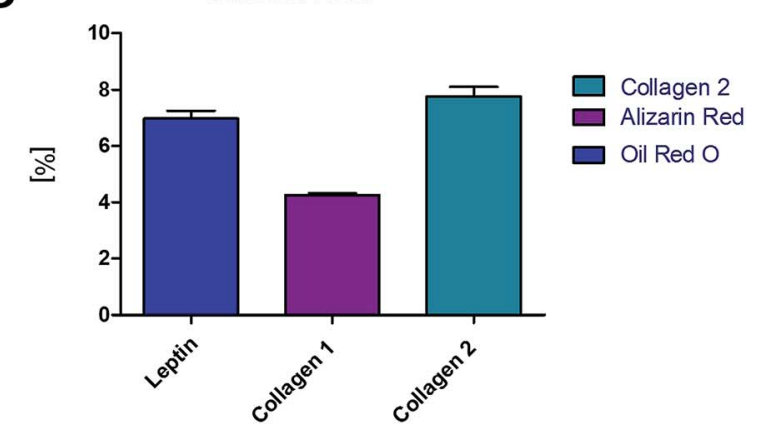

Fig. 6 Flow cytometry and multipotency analysis of isolated hASCs. Phenotype of hASCs was determined with flow cytometry. Isolated cells were strongly positive for CD44, CD73, CD90, and CD105 surface antigens. In contrast, the cells lacked the expression of hematopoietic markers CD45 and CD34 (A). Representative images from tri-lineage assay (B). Osteogenesis was confirmed with Alizarin red staining for extracellular matrix mineralizations, while proteoglycans formed during chondrogenesis were stained with Safranin O. The formation of intracellular lipidreached vacuoles as a typical characteristic of the adipogenic process was visualized with Oil Red O. Quantification of images shown as a percentage of the stained area with a certain dye (C). ELISA test results for chondrogenic, osteogenic, and adipogenic proteins (D). Magnification 100, scale bars $400 \mu \mathrm{m}$.

proliferative activity was noticed on the sample with $0.5 \mu \mathrm{mol}$ resveratrol, followed by the control sample without the addition of resveratrol (pure TPU/PUA) (Fig. 8A). The lowest proliferative activity was observed in the sample doped with $1 \mu \mathrm{mol}$ resveratrol. The shortest population doubling time was noticed in the sample with $0.5 \mu \mathrm{mol}$ resveratrol, while the longest time was in the sample with $1 \mu \mathrm{mol}$ resveratrol (Fig. 8B). Both results were significant in comparison to the control $(p<0.05)$.

The level of ROS detected was different in each investigated sample (Fig. 8C). The highest level of ROS was from samples doped with $1 \mu \mathrm{mol}$ resveratrol, whereas a significantly lower level of ROS was noticed in the sample with $0.5 \mu \mathrm{mol}$ resveratrol $(p<0.05)$ in relation to the pure control. Evaluation of NO concentrations in ASC revealed the highest level in the biomaterial with $1 \mu \mathrm{mol}$ resveratrol, and the lowest in the sample with $0.5 \mu \mathrm{mol}(p<0.05)$ (Fig. 8D). Measurements of SOD in the ASC population revealed its highest activity in the sample with 0.5 $\mu \mathrm{mol}(p<0.05)$, while in the other samples the changes were insignificant in comparison to the control (Fig. 8E).

\section{Detection of apoptosis- and autophagy-related genes}

In samples doped with $0.1 \mu \mathrm{mol}$ resveratrol, almost all the cells were viable and positively stained for calcein AM. The cells were elongated and created colonies connected with each other. Similarly, in the sample with $0.5 \mu \mathrm{mol}$ resveratrol, the cells were also mostly calcein-positive, with single dead cells present in 
D2

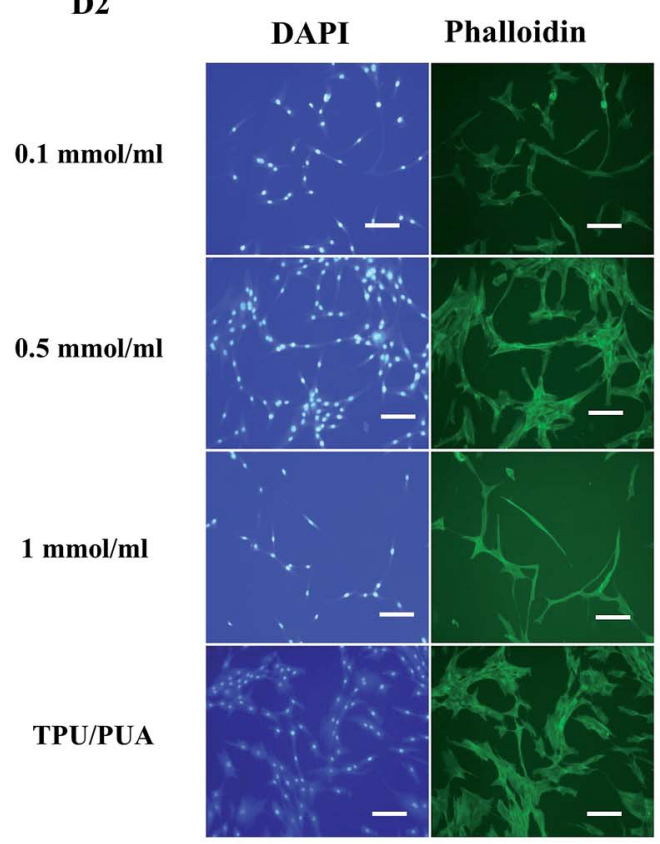

D5

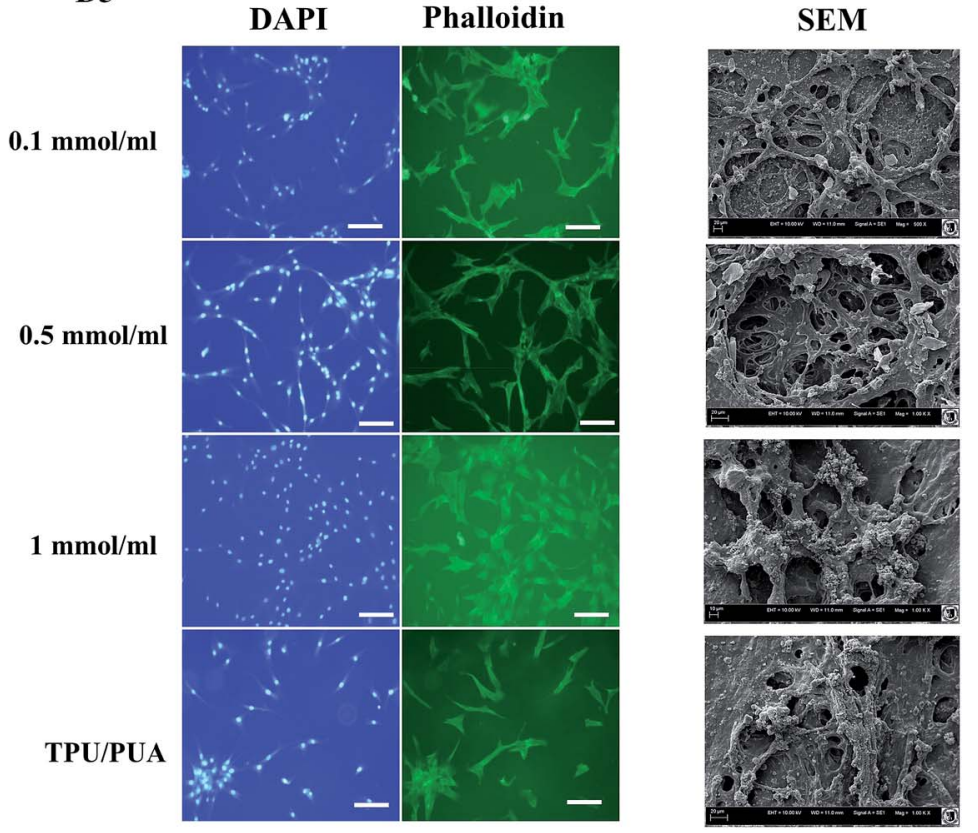

Fig. 7 ASCs cultured on experimental polymers stained histochemically for nuclei (DAPI), and actin (phalloidin), and film morphology visualized with SEM (fluorescence, magnification 100, scale bar $=200 \mu \mathrm{m}, \mathrm{D} 2=2 \mathrm{nd}$ day, D5 $=5$ th day of the experiment).

the monolayer (Fig. 9A). Cells were of elongated shape and formed clustered colonies connected with each other but grew mostly in an irregular pattern. Cells cultured on biomaterial with $1 \mu \mathrm{mol}$ resveratrol were mostly negative for calcein AM, and the propidium iodide staining revealed the presence of dead cells. Cells were mostly detached from the surface, while the remaining ones created elongated agglomerates. Furthermore, caspase- 3 positive cells was also observed in cells in all

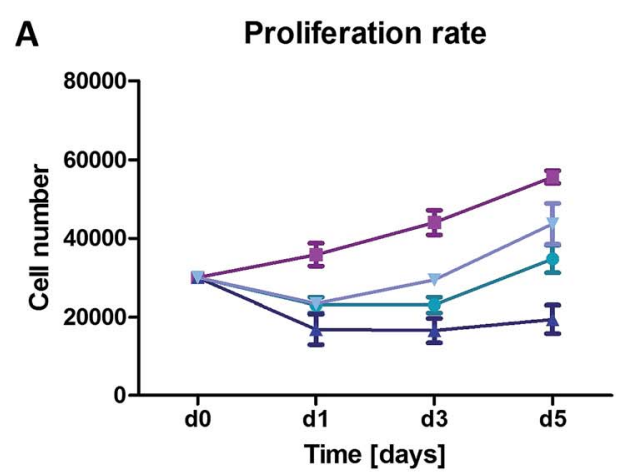

C

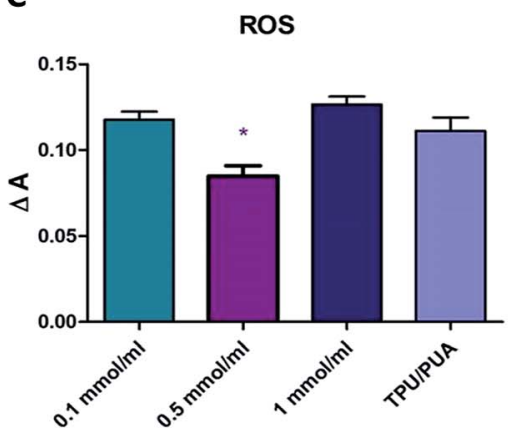

$\rightarrow 0.1 \mathrm{mmol} / \mathrm{ml}$

- $0.5 \mathrm{mmol} / \mathrm{ml}$

$\pm 1 \mathrm{mmol} / \mathrm{ml}$

$\rightarrow$ TPU/PUA
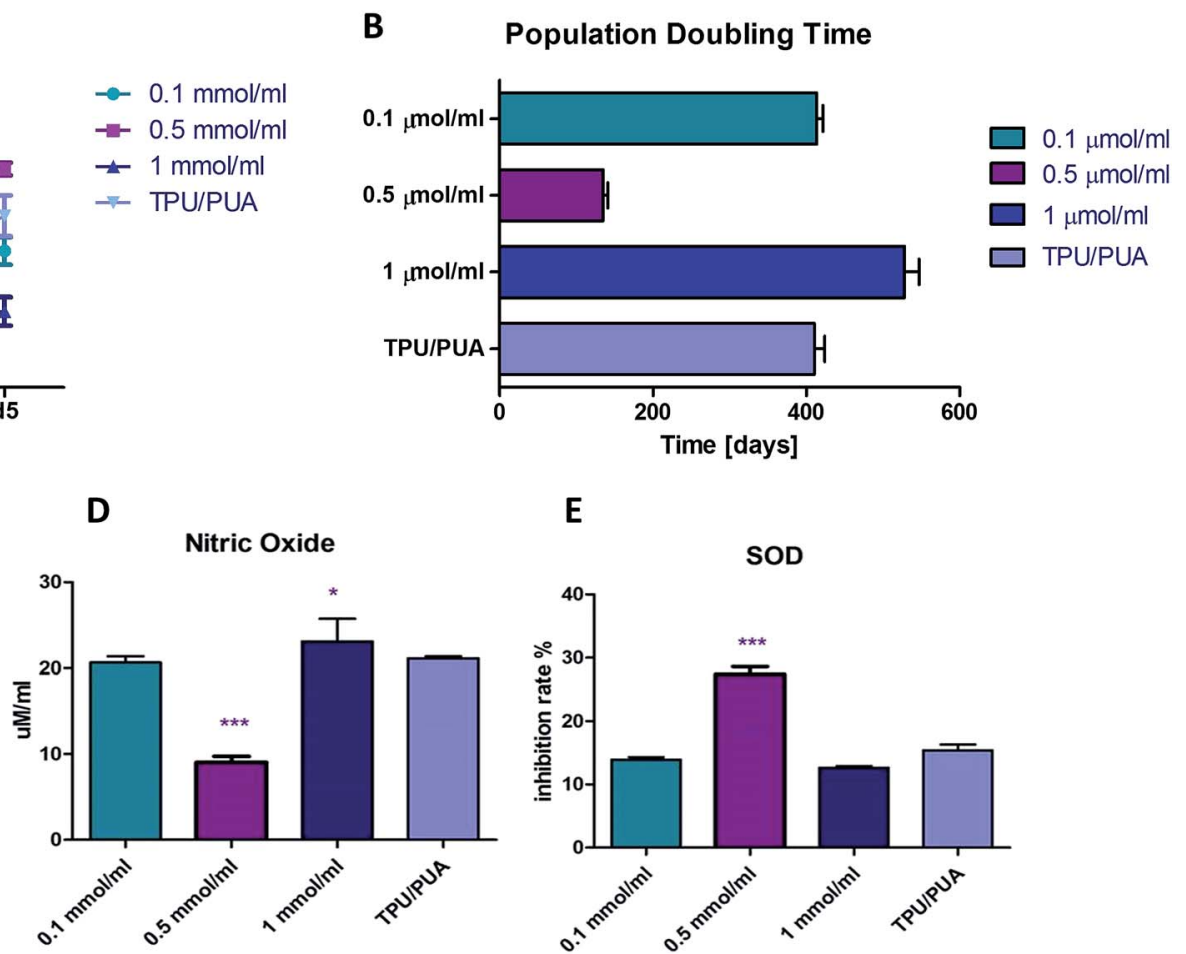

$\mathbf{E}$

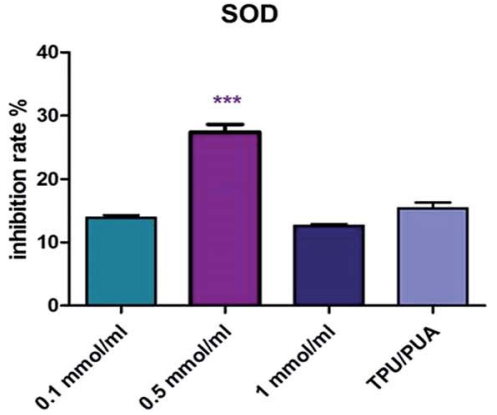

Fig. 8 Growth curves and the population doubling times of adipose stem cells during five days of experimental culture on different polymers; the quantity of reactive oxygen species, nitric oxide, and superoxide dismutase in adipose stem cells cultured on all the tested biomaterials $(* p<0.05$, $* * p<0.01, * * * p<0.001)$. 
A

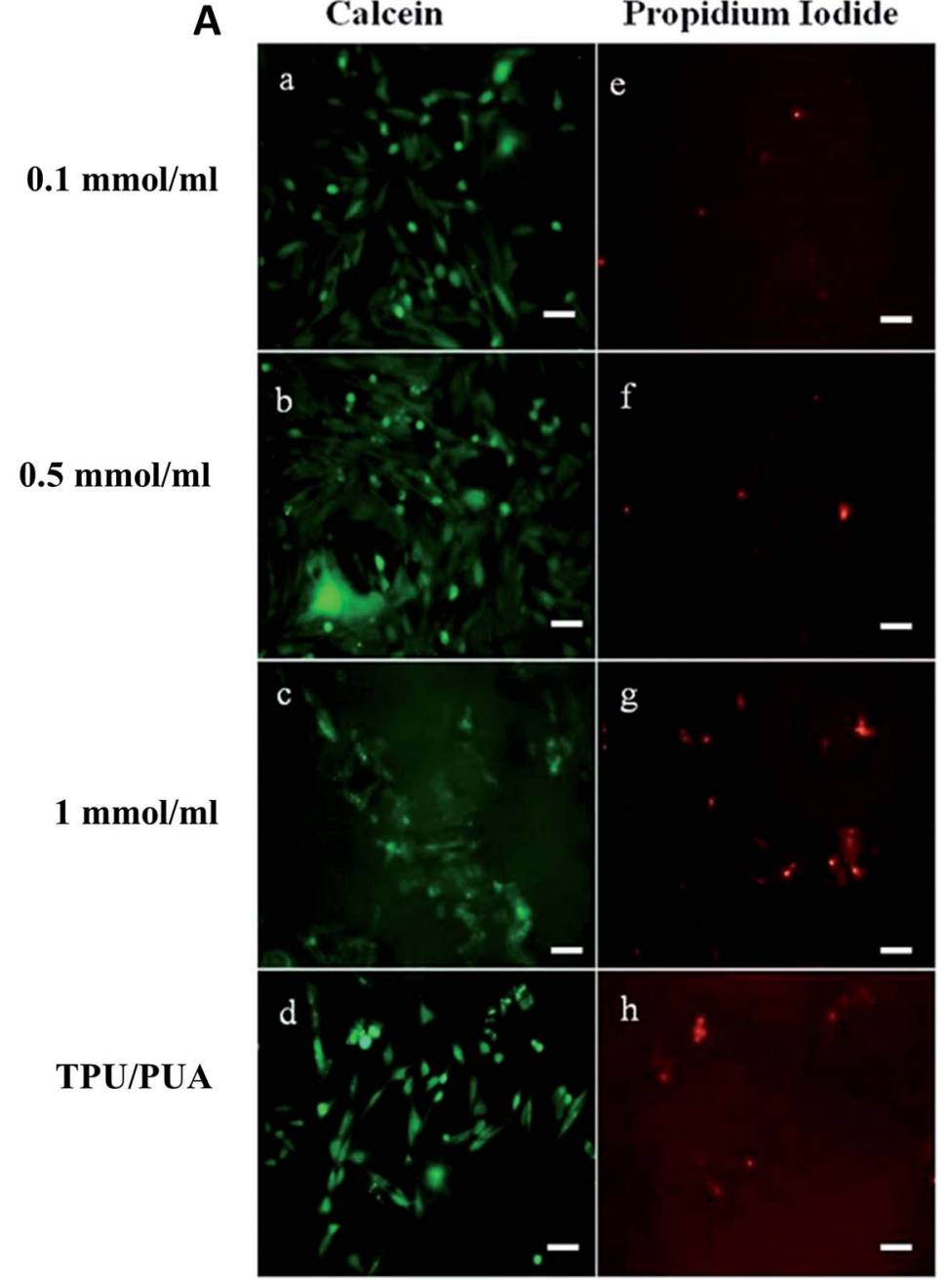

Propidium Iodide
B

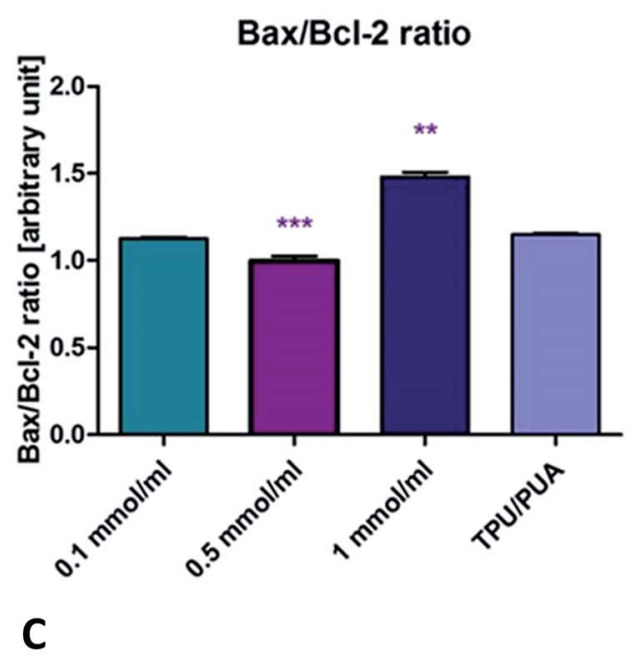

p53/p21 ratio

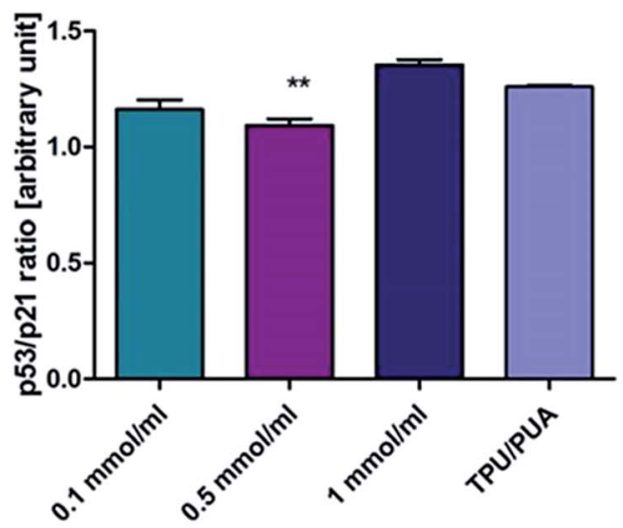

Fig. 9 The calcein/propidium iodide staining of adipose stem cells cultured on different polymers; green - live, red - dead; magnification 100, scale bar $=200 \mu \mathrm{m}$. The real-time PCR results of mRNA level apoptosis-related gene ratios * - Significantly different from the control $(* p<0.05$, $* * p<0.01, * * * p<0.001)$.

the investigated groups. Analysis of the apoptosis-related genes: Bax, Bcl-2, p21, and also tumor suppressor p53 gene expression was carried out in ASCs cultured on biomaterials doped with resveratrol (Fig. 9B and C). Quantitative analysis of the transcripts revealed that the $\mathrm{Bax} / \mathrm{Bcl}-2$ and $\mathrm{p} 53 / \mathrm{p} 21$ ratio was significantly decreased in the $0.5 \mu \mathrm{mol}$ cultures. What is interesting is that the level of the $\mathrm{Bax} / \mathrm{Bcl}-2$ transcripts ratio was also significantly increased in cells cultured on $1 \mu \mathrm{mol}$ resveratroldoped biomaterial $(p<0.05)$.

Moreover the morphology of the mitochondria varied depending on the cell type and activity on the biomaterials. At $0.5 \mu \mathrm{mol}$ resveratrol, the doped biomaterial and pure biomaterial cells' mitochondria were organized in a dynamic network with a multi-branched structure. In the $0.1 \mu \mathrm{mol}$ and $1 \mu \mathrm{mol}$ resveratrol-doped groups, we observed a disrupted mitochondrial distribution (Fig. 10A).

Moreover analysis of the autophagy-related genes in cells cultured on biomaterials doped with resveratrol indicated that $0.1,0.5$, and $1 \mu \mathrm{mol}$ resveratrol significantly down-regulated CHOP (Fig. 10B) and Beclin-1 (Fig. 10C) mRNA levels in ASCs in comparison to the control. In accordance with the changes observed in CHOP and Beclin-1 mRNA, BPINK and Parkin genes expression was studied. Significantly higher mRNA copy number for both Pink (Fig. 10D) and Parkin (Fig. 10E) was confirmed in the ASC cultured on biomaterials doped with 0.1 and $0.5 \mu \mathrm{mol}$ resveratrol.

\section{Chondrogenic and osteogenic differentiation induction potential of biomaterials doped with resveratrol}

Analysis of gene expression in cells cultured on biomaterials doped with 0.1 and $0.5 \mu \mathrm{mol}$ resveratrol showed an upregulation of a range of markers characteristic of osteogenic differentiation and genes encoding extracellular matrix molecules expressed in chondrogenic differentiation. The $0.1,0.5$, and 1 $\mu \mathrm{mol}$ additions of resveratrol caused significant upregulation of BMP-2 (Fig. 11A) in ASCs cultured on biomaterials in comparison to pure TPU/PUA. Furthermore, cells cultured on biomaterials with 0.1 and $0.5 \mu \mathrm{mol}$ resveratrol additions were characterized by significantly upregulated osteocalcin 
A
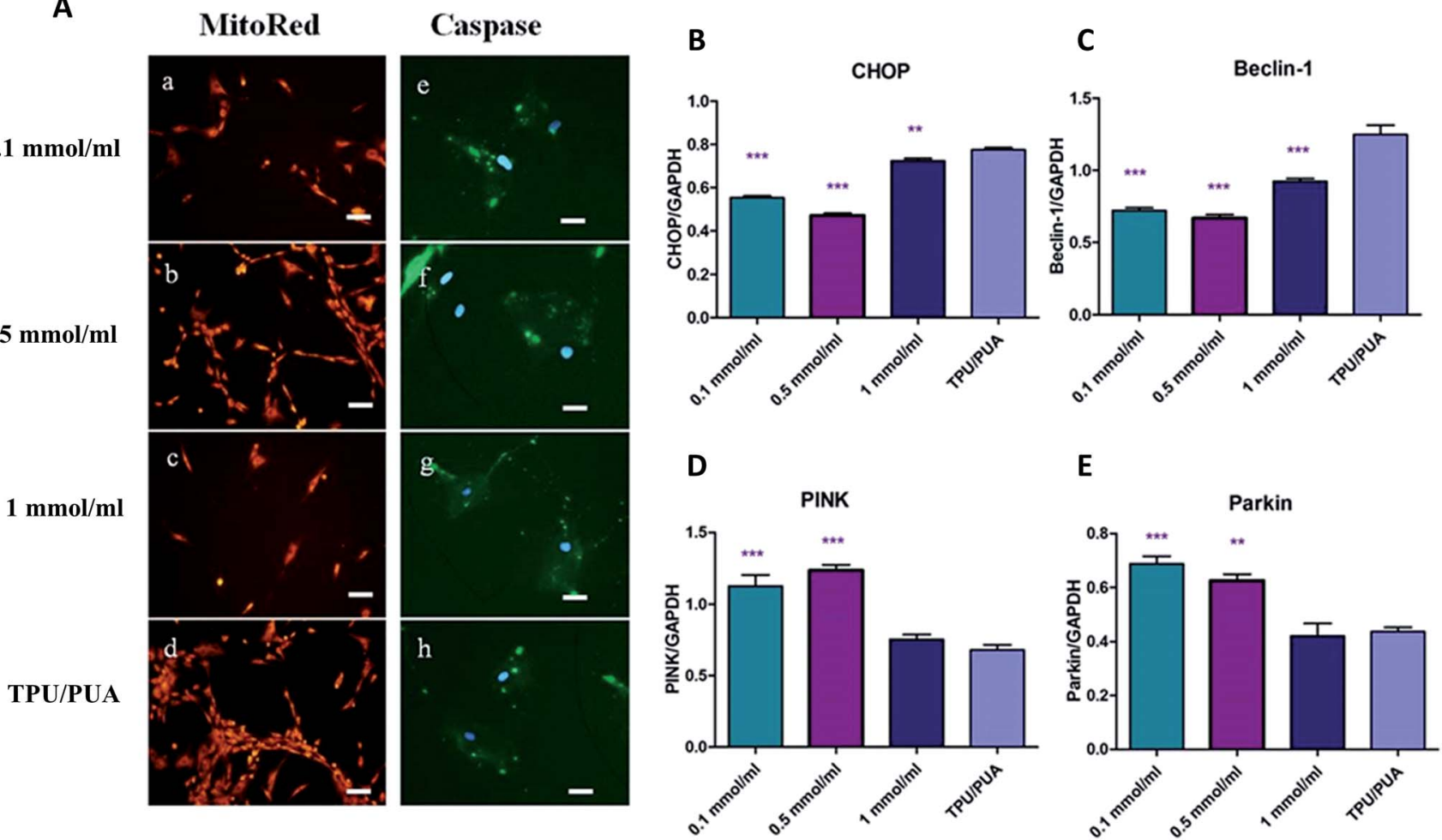

Fig. 10 Images showing mitochondria stained with MitoRed dye and the accumulation of caspase. The real-time PCR results $(* p<0.05$, ** $p<$ $\left.0.01,{ }^{* * *} p<0.001\right)$; magnification 100 , scale bar $=200 \mu \mathrm{m}$.

A

BMP-2

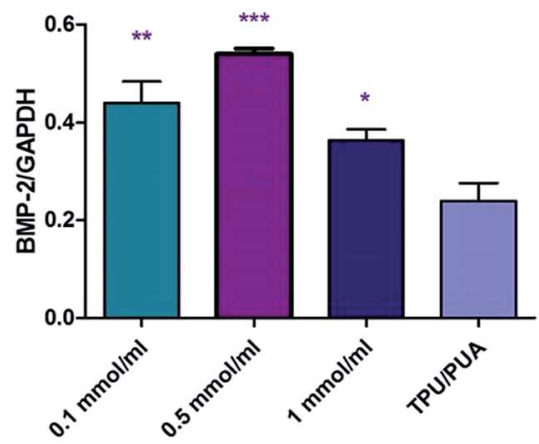

D

Aggrecan

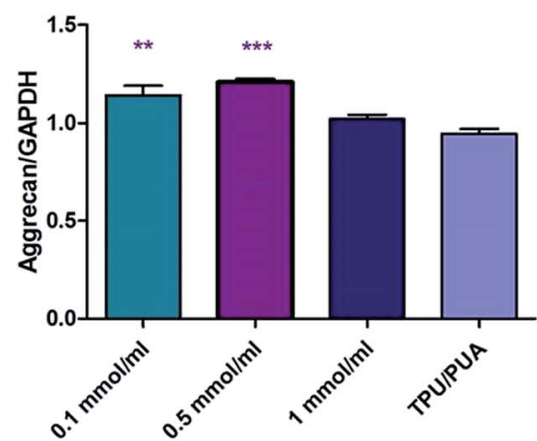

B

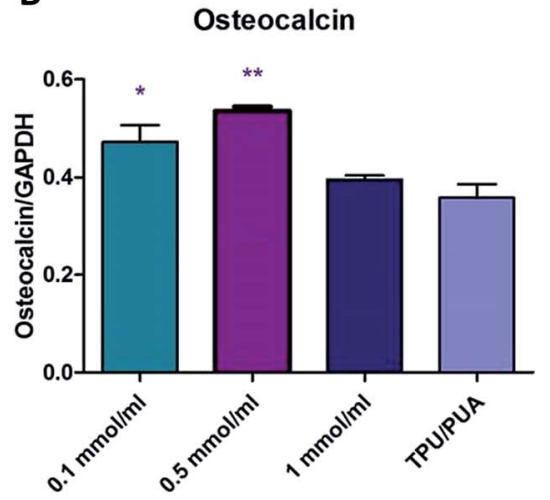

E

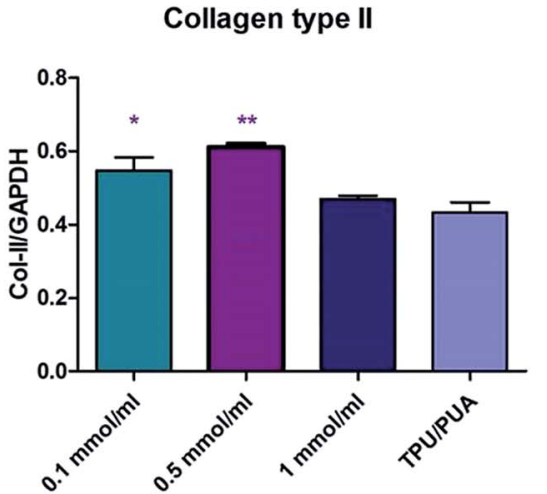

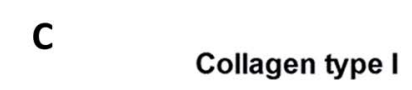
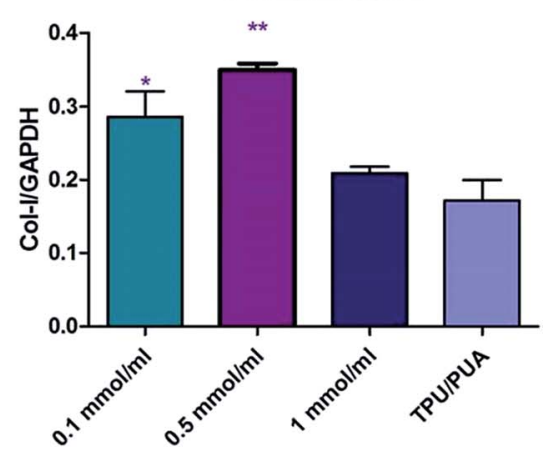

$\mathbf{F}$

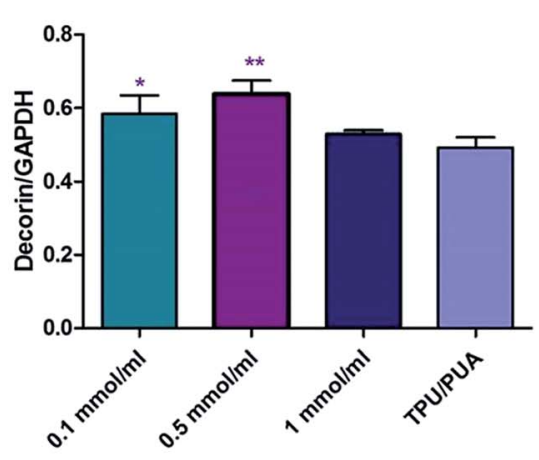

Fig. 11 The real-time RT-PCR results obtained from ASCs cultured on different biomaterials showing different levels of mRNA expression genes responsible for osteogenic and chondrogenic differentiation $(* p<0.05, * * p<0.01, * * * p<0.001)$. 
(Fig. 11B), collagen type I (Fig. 11C) and II (Fig. 11D), aggrecan (Fig. 11E), and decorin (Fig. 11F) gene expression.

\section{Discussion}

Resveratrol (RES) is a natural, plant-derived antioxidant that serves as an agonist for the estrogen receptor, promoting estrogen-like effects, such as neuroprotection and bone growth. Supplementation with estrogen is generally used to decrease bone loss by enhancing osteoblast activity in skeletal diseases. Thus, resveratrol supplementation may help to regenerate the bone mass, especially in an inflammatory microenvironment characteristic for bone fractures.

Although supplementation of culture media with different doses of resveratrol and its influence on cellular metabolism have been well studied and described in the literature, the fabrication of resveratrol-doped biomaterials for tissue engineering is an emerging field. In the present study, we decided to engineer an antioxidative, osteoinductive scaffold that could harness the proliferative potential of ASCs, as well as program them toward bone tissue formation. To accomplish this goal, we cultured ASC in the presence of TPU/PLA polymers doped with different concentrations of RES and established their growth kinetics, oxidative stress markers, and gene expression profile. The developed novel scaffold stands as an innovative approach to enhance graft integration and assimilation with native tissue.

The study conducted by Kleinedler et $a .^{24}$ describes the development and evaluation of polymers that provide a release of vascular-protective polyphenols for endovascular devices. RES combined with querencin was incorporated into an arborescent poly(styrene-isobutylene-styrene) tri-block polymer (arbIBS) and applied to stainless steel coupons using an electrospray process. The obtained results indicated that arbIBS exhibits no cytotoxicity, and that the films released the mentioned substances at therapeutic levels, also dosedependently inhibiting macrophage activation, VSMC proliferation, and platelet stimulation.

Because of its unique properties, including antibacterial, antifungal, and anti-inflammatory properties, RES was also used in the study conducted by Pinho et al. ${ }^{25}$ for the generation of natural and environmental friendly materials. Their work showed that it is possible to obtain cotton, bamboo, and silk functionalized with RES. The huge limitation of this study was its biological part as they only performed one in vivo cytotoxicity assay using 3T3 fibroblast cells without any more sophisticated tests. The material and chemical part of the study was more robust, giving details about the fabrics characteristics, including RES release. A prolonged release of bioactive molecules may overcome the adverse effects elicited by the therapeutic delivery of certain indigenous growth factors. Moreover, it is crucial for an effective, biofunctional material with pharmacological properties to release active molecules in a controlled manner. Fabricated by our group, TPU/PLA/RES films are characterized by a prolonged RES release as we observed RES desorption for up to 40 days. It is worth noting that the highest rate of RES release lasted till day 20, while after up to 40 days it stayed almost at a constant level. This is a valuable feature when taking into consideration the biomedical application of our scaffold. As it was shown in a rat model of postoperative pain, the tissue concentrations of IL-1beta, IL-6, CINC-1, and TNF-alpha were greatly increased within $24-48 \mathrm{~h}$ of surgery and remain elevated for up to 8 days. ${ }^{26}$ Thus, the increased RES release during the first 20 days may provide beneficial effect in tissue regeneration and reduce existing inflammation. The proinflammatory cytokine release profile was also established for in humans by Kumulosasi et al., who observed the highest expression of IL-1, IL-8, and TNF-alpha during the 1st day of inflammation. To conclude, a prolonged release of RES from biomaterials is highly desirable in bone tissue engineering because of its osteoconductive, osteoinductive, and anti-inflammatory properties. ${ }^{27}$

Other aspects to consider are the influence of different temperatures on the scaffolds and the biodegradation time. Taking into consideration the in vivo application of the fabricated scaffold, it is crucial to establish the time of its biodegradation at physiological temperature. Our results indicate that $40 \%$ of RES-enriched TPU/PLA films degraded after 20 weeks in water in $37{ }^{\circ} \mathrm{C}$; however, this was in vitro. Further studies are required to fully describe the behavior and degradation time of the presented material in vivo. For a clinical point of view, it is crucial to evaluate whether the biomaterial can be sterilized and if high temperatures can change its characteristics. The fabricated TPU/PLA/RES scaffold could retain its mass and composition in temperatures between $80^{\circ} \mathrm{C}$ and $260^{\circ} \mathrm{C}$. This will allow for sterilization of the scaffold in dry heat sterilizers and its safe usage in clinical practice without losing its unique properties.

Biomaterial enriched with resveratrol was mainly created for targeting in bone tissue regeneration, especially in elderly patients, as they comprise a major group suffering from musculoskeletal disorders. The use of RES as a osteogenesis enhancer has been previously investigated in MSC and MC3T3E1 cells. ${ }^{28}$ It was also proved that RES affects the cells in a timeand dose-dependent manner. ASCs cultured with $12.5 \mu \mathrm{M}, 25$ $\mu \mathrm{M}$, and $50 \mu \mathrm{M}$ resveratrol showed the highest proliferation and the highest levels of ALP when cultured with $25 \mu \mathrm{M}$ resveratrol. Doses of $50 \mu \mathrm{M}$ resulted in extremely low cell numbers and ALP production..$^{28}$ Another study concluded that doses higher than $25 \mu \mathrm{M}$ resveratrol are potentially cytotoxic..$^{29}$ Based on different studies, we decided to prepare scaffolds with three different RES concentration. Our results indicated that even a slight change in RES significantly affected the ASC population. Based on the proliferation rate of ASC, we established that $0.5 \mathrm{mmol} \mathrm{ml} l^{-1}$ is the most effective RES working concentration, while $1 \mathrm{mmol}$ $\mathrm{ml}^{-1}$ displayed a cytotoxic effect.

Bearing in mind the fact that age negatively affects ASC features, including an excessive accumulation of ROS, decreased activity of antioxidative enzymes, and mitochondria deterioration, ${ }^{5,30}$ we investigated whether the fabricated scaffold possessed anti-aging and mitochondria protective properties. Excessive ROS accumulation may induce the oxidative modification of cellular macromolecules, including lipid and protein oxidation. One of the biological activities ascribed to resveratrol involves its antioxidant potential. Resveratrol is both a free radical scavenger and a potent antioxidant because of its ability 
to promote the activities of a variety of antioxidant enzymes. ${ }^{31}$ Studies have confirmed that RES can increase the amounts of SOD, glutathione peroxidase, glutathione $S$-transferase, and glutathione reductase. ${ }^{32}$ Our data stands in good agreement with the above data as ASC cultured on TPU/PLA film doped with $0.5 \mathrm{mmol} \mathrm{ml} \mathrm{m}^{-1}$ RES presented a decreased accumulation of ROS and NO, while the activity of SOD was significantly increased in comparison to pure TPU/PLA film.

Besides its antioxidative properties, RES is also known as an anti- and pro-apoptotic agent. A study by Huang et $a .^{33}$ revealed that pretreatment with RES prevented ethanol-induced disruption of embryonic development in vitro and in vivo. Moreover, RSE attenuated $\beta$-amyloid-induced cytotoxicity and apoptotic features in PC12 cells. On the other hand, RES was proved to induce apoptosis in several cancer cell lines. ${ }^{34,35}$ Our results indicated anti-apoptotic characteristics of the $0.5 \mathrm{mmol} \mathrm{ml} \mathrm{m}^{-1}$ TPU/PLA/RES scaffold as we observed an increased number of dead cells and decreased ratios of Bcl-2/Bax, p53, and p21 genes expression, which are known as apoptotic markers.

It was also proved that RES was able to modulate mitochondrial dynamics mainly by inducing mitochondrial biogenesis and protect against metabolic decline. SIRT1 plays an essential role in the ability of different doses of resveratrol to stimulate AMPK and to improve the mitochondrial function both in vitro and in vivo. Interestingly, in our study we observed that RES increased the expression of Pink and Parkin genes, both related to the mitophagy process. The role of RES in autophagy is a well-established phenomenon as RES inhibits mTOR activity and in consequence autophagy induction. ${ }^{36}$ That feature of RES has been proven in various cell lines and model organisms, such as Caenorhabditis elegans and mice. ${ }^{37}$ Our data indicated that RES may also contribute to a clearance of dysfunctional mitochondria, which are prone to an accumulation of excessive ROS by the mitophagy induction. This is especially important in the case of geriatric regenerative medicine as ASC isolated from elderly are characterized by mitochondria deterioration. ${ }^{5}$

Endoplasmic reticulum (ER) stress leads to an evolutionary conserved stress response called the unfolded protein response (UPR). The main purpose of UPR is to compensate for damage, although when the ER dysfunction is severe or prolonged it can trigger apoptosis. ${ }^{6}$ The excessive production and accumulation of ROS has been linked to both ER stress and UPR. Although ROS play a critical role in many cellular processes, its robust amount contributes to harmful effects, including protein misfolding and oxidation, which leads to apoptotic death and contributes to various degenerative diseases. Some UPR components, such as the C/EBP homologous protein CHOP, can contribute to oxidative stress and also in in vitro and animals studies demonstrated that $\mathrm{CHOP}$ is a master regulator of ER stress-induced apoptosis. ${ }^{38}$ In neuronal cells, CHOP may transactivate the p53 upregulated modulator of apoptosis (PUMA) on ER stress through cooperation with the transcription factor forkhead box, class $\mathrm{O}, 3 \mathrm{a} \cdot{ }^{39}$ Moreover it can also inhibit the pro-survival protein $\mathrm{Bcl}-2$ through transcriptional suppression, which may require the liver inhibitory protein, an isoform of $\mathrm{C} / \mathrm{EBP} \beta .^{40}$ Studies also implicated a role for CHOP in promoting protein synthesis to cause oxidative stress, leading to apoptosis. ${ }^{41}$ Aging-linked declines in the expression and activity of the key ER molecular chaperones and folding enzymes compromise proper protein folding and the adaptive response of the UPR. ${ }^{42}$ Fabricated by our group, the biomaterial doped with $0.5 \mathrm{mmol} \mathrm{m} \mathrm{m}^{-1}$ RES significantly downregulated the expression of CHOP in human ASC. Thus its usage in the therapy of elderly patients seems to be even more reasonable as ER stress increases with age and disease. Our data stands in good agreement with Graham et al., who proposed that RES augments ER stress and the cytotoxic effects of glycolytic inhibition in neuroblastoma by downregulating Akt. ${ }^{43}$

As it was shown in multiple research studies, RES works in multiple ways, activating different cellular metabolism pathways. It was also shown that RES is able to induce osteogenesis, although the mechanism of its action is still under investigation. Preliminary data have shown that RES triggers the Wnt signaling pathway leading to the upregulation of RUNX2 expressionessential for cell differentiation into osteoblasts. ${ }^{44}$ Moreover, it promotes osteogenesis through SIRT-1, whose activation operates synergistically to mediate RUNX2 gene transcription. ${ }^{20}$ In our study, we observed that TPU/PLA films doped with $0.5 \mathrm{mmol}$ $\mathrm{ml}^{-1}$ RES acted as stimulators of osteogenesis as we observed the upregulation of osteogenesis-related genes in human ASC cultured on that scaffold. Our fabricated material directed ASC toward an osteoblast-like molecular phenotype, which makes it a promising tool in regenerative medicine. The combination of a scaffold with ASC may improve the effectiveness and shorten the time of bone regeneration. Among the investigated TPU/PLA scaffolds, the one doped with $0.5 \mathrm{mmol} \mathrm{ml}^{-1} \mathrm{RES}$ resulted in the greatest osteogenesis-inducing potential, as we observed a significantly increased expression of the following genes: BMP2, osteocalcin, collagen type I, aggrecan, collagen type II, and decorin, all of which are crucial for the initiation of osteogenesis and bone formation. However, further studies are need to confirm that data and elucidate the effect of RES doped TPU/PLA biomaterials on osteogenic signaling pathways using both, in vitro and in vivo models.

To conclude, in the present study we fabricated innovative biomaterials characterized by RES release in a sustained manner. The prolonged release of RES is especially important when considering its clinical application. Moreover, the release of RES was most intense during the first 20 days, which may be beneficial in vivo as inflammation within damaged tissue is the most intense during the first days after injury. Moreover, among the tested scaffolds, TPU/PLA doped with $0.5 \mathrm{mmol} \mathrm{ml} \mathrm{m}^{-1}$ RES was the most potent in increasing proliferation, attenuating apoptosis, oxidative, and ER stress, and in the induction of osteogenesis in human ASC. These characteristics make the biomaterial presented here a promising tool in the emerging field of personalized regenerative medicine, especially in geriatrics.

\section{Acknowledgements}

Material part of study including preparation of scaffold was supported by National Science Centre, Poland grant no. 2015/ 
19/B/ST5/01330. Biological part of research was supported by National Science Centre, Poland grant no. 015/18/E/NZ9/0060 and National Science Centre, Poland grant no. 2016/21/B/NZ7/ 01111. Publication supported by Wrocław Centre of Biotechnology, programme the Leading National Research Centre (KNOW) for years 2014-2018.

\section{References}

1 S. Geißler, M. Textor, J. Kühnisch, D. Könnig, O. Klein, A. Ode, T. Pfitzner, J. Adjaye, G. Kasper and G. N. Duda, PLoS One, 2012, 7, e52700.

2 K. Marycz, J. Krzak-Roś, A. Donesz-Sikorska and A. Śmieszek, J. Biomed. Mater. Res., Part A, 2014, 102, 4017-4026.

3 K. Marycz, D. Szarek, J. Grzesiak and K. Wrzeszcz, Biomed. Mater. Eng., 2014, 24, 1625-1637.

4 R. Starosta, A. Brzuszkiewicz, A. Bykowska, U. K. Komarnicka, B. Bażanów, M. Florek, Ł. Gadzała, N. Jackulak, J. Król and K. Marycz, Polyhedron, 2013, 50, 481-489.

5 K. Kornicka, K. Marycz, K. A. Tomaszewski, M. Marędziak and A. Śmieszek, Oxid. Med. Cell. Longevity, 2015, 2015, 309169.

6 C. Xu, B. Bailly-Maitre and J. C. Reed, J. Clin. Invest., 2005, 115, 2656-2664.

7 D. Baksh, R. Yao and R. S. Tuan, Stem Cells, 2007, 25, 13841392.

8 A. Stolzing and A. Scutt, Aging Cell, 2006, 5, 213-224.

9 A. Stolzing, E. Jones, D. McGonagle and A. Scutt, Mech. Ageing Dev., 2008, 129, 163-173.

10 M. Z. Ratajczak, K. Marycz, A. Poniewierska-Baran, K. Fiedorowicz, M. Zbucka-Kretowska and M. Moniuszko, Adv. Med. Sci., 2014, 59, 273-280.

11 K. Nishimura and J. Takahashi, Biol. Pharm. Bull., 2013, 36, 171-175.

12 B. Larijani, E. N. Esfahani, P. Amini, B. Nikbin, K. Alimoghaddam, S. Amiri, R. Malekzadeh, N. M. Yazdi, M. Ghodsi, Y. Dowlati, M. A. Sahraian and A. Ghavamzadeh, Acta Med. Iran., 2012, 50, 79-96.

13 X. Gao, Y. X. Xu, N. Janakiraman, R. A. Chapman and S. C. Gautam, Biochem. Pharmacol., 2001, 62, 1299-1308.

14 J. A. Baur, K. J. Pearson, N. L. Price, H. A. Jamieson, C. Lerin, A. Kalra, V. V. Prabhu, J. S. Allard, G. Lopez-Lluch, K. Lewis, P. J. Pistell, S. Poosala, K. G. Becker, O. Boss, D. Gwinn, M. Wang, S. Ramaswamy, K. W. Fishbein, R. G. Spencer, E. G. Lakatta, D. Le Couteur, R. J. Shaw, P. Navas, P. Puigserver, D. K. Ingram, R. de Cabo and D. A. Sinclair, Nature, 2006, 444, 337-342.

15 K. J. Pearson, J. A. Baur, K. N. Lewis, L. Peshkin, N. L. Price, N. Labinskyy, W. R. Swindell, D. Kamara, R. K. Minor, E. Perez, H. A. Jamieson, Y. Zhang, S. R. Dunn, K. Sharma, N. Pleshko, L. A. Woollett, A. Csiszar, Y. Ikeno, D. Le Couteur, P. J. Elliott, K. G. Becker, P. Navas, D. K. Ingram, N. S. Wolf, Z. Ungvari, D. A. Sinclair and R. de Cabo, Cell Metab., 2008, 8, 157-168.

16 J. G. Wood, B. Rogina, S. Lavu, K. Howitz, S. L. Helfand, M. Tatar and D. Sinclair, Nature, 2004, 430, 686-689.
17 J. C. Tou, Biochim. Biophys. Acta, 2015, 1852, 1186-1194.

18 D. Vergara, P. Simeone, D. Toraldo, P. Del Boccio, V. Vergaro, S. Leporatti, D. Pieragostino, A. Tinelli, S. De Domenico, S. Alberti, A. Urbani, M. Salzet, A. Santino and M. Maffia, Mol. BioSyst., 2012, 8, 1078-1087.

19 P.-C. Tseng, S.-M. Hou, R.-J. Chen, H.-W. Peng, C.-F. Hsieh, M.-L. Kuo and M.-L. Yen, J. Bone Miner. Res., 2011, 26, 2552-2563.

20 M. Shakibaei, P. Shayan, F. Busch, C. Aldinger, C. Buhrmann, C. Lueders and A. Mobasheri, PLoS One, 2012, 7, e35712.

21 C.-L. Kao, L.-K. Tai, S.-H. Chiou, Y.-J. Chen, K.-H. Lee, S.-J. Chou, Y.-L. Chang, C.-M. Chang, S.-J. Chen, H.-H. Ku and H.-Y. Li, Stem Cells Dev., 2010, 19, 247-258.

22 D. Szarek, K. Marycz, J. Laska, P. Bednarz and W. Jarmundowicz, Scanning, 2013, 35, 232-245.

23 J. Grzesiak, K. Marycz, D. Szarek, P. Bednarz and J. Laska, Mater. Sci. Eng., C, 2015, 52, 163-170.

24 J. J. Kleinedler, J. D. Foley, J. S. Alexander, S. C. Roerig, V. Y. Hebert and T. R. Dugas, J. Biomed. Mater. Res., Part B, 2011, 99, 266-275.

25 E. Pinho, M. Henriques, R. Oliveira, A. Dias and G. Soares, Fibers Polym., 2010, 11, 271-276.

26 L. C. Loram, A. C. Themistocleous, L. G. Fick and P. R. Kamerman, Can. J. Physiol. Pharmacol., 2007, 85, 613620.

27 M. S. Kamath, S. S. Ahmed, M. Dhanasekaran and S. W. Santosh, Int. J. Nanomed., 2013, 9, 183-195.

28 C. P. Erdman, C. R. Dosier, R. Olivares-Navarrete, C. Baile, R. E. Guldberg, Z. Schwartz and B. D. Boyan, J. Tissue Eng. Regener. Med., 2012, 6(suppl. 3), s34-46.

29 C. R. Dosier, C. P. Erdman, J. H. Park, Z. Schwartz, B. D. Boyan and R. E. Guldberg, J. Mech. Behav. Biomed. Mater., 2012, 11, 112-122.

30 K. Kornicka, B. Babiarczuk, J. Krzak and K. Marycz, RSC Adv., 2016, 6, 29524-29537.

31 R. Zini, C. Morin, A. Bertelli, A. A. Bertelli and J. P. Tillement, Drugs Exp. Clin. Res., 1999, 25, 87-97.

32 G.-C. Yen, P.-D. Duh and C.-W. Lin, Free Radical Res., 2003, 37, 509-514.

33 L.-H. Huang, N.-H. Shiao, Y.-D. Hsuuw and W.-H. Chan, Toxicology, 2007, 242, 109-122.

34 Y.-J. Surh, Y.-J. Hurh, J.-Y. Kang, E. Lee, G. Kong and S. J. Lee, Cancer Lett., 1999, 140, 1-10.

35 T. Hsieh and J. M. Wu, Exp. Cell Res., 1999, 249, 109-115.

36 D. Park, H. Jeong, M. N. Lee, A. Koh, O. Kwon, Y. R. Yang, J. Noh, P.-G. Suh, H. Park and S. H. Ryu, Sci. Rep., 2016, 6, 21772.

37 A. Puissant, G. Robert, N. Fenouille, F. Luciano, J.-P. Cassuto, S. Raynaud and P. Auberger, Cancer Res., 2010, 70, 1042-1052.

38 D. T. Rutkowski, S. M. Arnold, C. N. Miller, J. Wu, J. Li, K. M. Gunnison, K. Mori, A. A. Sadighi Akha, D. Raden and R. J. Kaufman, PLoS Biol., 2006, 4, e374.

39 A. P. Ghosh, B. J. Klocke, M. E. Ballestas and K. A. Roth, PLoS One, 2012, 7, e39586. 
40 C.-B. Chiribau, F. Gaccioli, C. C. Huang, C. L. Yuan and M. Hatzoglou, Mol. Cell. Biol., 2010, 30, 3722-3731.

41 S. J. Marciniak, C. Y. Yun, S. Oyadomari, I. Novoa, Y. Zhang, R. Jungreis, K. Nagata, H. P. Harding and D. Ron, Genes Dev., 2004, 18, 3066-3077.

42 M. K. Brown and N. Naidoo, Front. Physiol., 2012, 3, 263.
43 R. M. Graham, F. Hernandez, N. Puerta, G. De Angulo, K. A. Webster and S. Vanni, Exp. Mol. Med., 2016, 48, e210. 44 H. Zhou, L. Shang, X. Li, X. Zhang, G. Gao, C. Guo, B. Chen, Q. Liu, Y. Gong and C. Shao, Exp. Cell Res., 2009, 315, 29532962. 